\title{
Multiresolution Molecular Mechanics: Surface effects in nanoscale materials
}

\author{
Qingcheng Yang ${ }^{\dagger}$, Albert C. To ${ }^{*}$ \\ Department of Mechanical Engineering and Materials Science, University of Pittsburgh, \\ Pittsburgh, Pennsylvania, USA
}

First author: Qingcheng Yang, 636 Benedum Hall, University of Pittsburgh, Pittsburgh, Pennsylvania, 15261, Email: qiy9@ pitt.edu

${ }^{\dagger}$ Present Address: N257 Millenium Science Complex, Department of Materials Science and

Engineering, Penn State University, University Park, Pennsylvania, 16802; Email:

$$
\text { qzy25@psu.edu }
$$

*Corresponding Author: Albert C. To, 508 Benedum Hall, University of Pittsburgh, Pennsylvania 15261, Tel: (412) 624-2052; Email: albertto@pitt.edu 


\begin{abstract}
Surface effects have been observed to contribute significantly to the mechanical response of nanoscale structures. The newly proposed energy-based coarse-grained atomistic method Multiresolution Molecular Mechanics (MMM) [Q. Yang, A.C. To, Comput. Methods in Appl. Mech. Eng. 283 (2015) 384-418] is applied to capture surface effect for nanosized structures by designing a surface summation rule $\mathrm{SR}^{\mathrm{S}}$ within the framework of MMM. Combined with previously proposed bulk summation rule $\mathrm{SR}^{\mathrm{B}}$, the $\mathrm{MMM}$ summation rule $\mathrm{SR}^{\mathrm{MMM}}$ is completed. $\mathrm{SR}^{\mathrm{S}}$ and $\mathrm{SR}^{\mathrm{B}}$ are consistently formed within $\mathrm{SR}^{\mathrm{MMM}}$ for general finite element shape functions. Analogous to quadrature rules in finite element method (FEM), the key idea to the good performance of $\mathrm{SR}^{\mathrm{MMM}}$ lies in that the order or distribution of energy for coarse-grained atomistic model is mathematically derived such that the number, position and weight of quadrature-type (sampling) atoms can be determined. Mathematically, the derived energy distribution of surface area is different from that of bulk region. Physically, the difference is due to the fact that surface atoms lack neighboring bonding. As such, $\mathrm{SR}^{\mathrm{S}}$ and $\mathrm{SR}^{\mathrm{B}}$ are employed for surface and bulk domains, respectively. Two- and three-dimensional numerical examples using the respective 4-node bilinear quadrilateral, 8-node quadratic quadrilateral and 8-node hexahedral meshes are employed to verify and validate the proposed approach. It is shown that MMM with $\mathrm{SR}^{\mathrm{MMM}}$ accurately captures corner, edge and surface effects with less $0.3 \%$ degrees of freedom of the original atomistic system, compared against full atomistic simulation. The effectiveness of $\mathrm{SR}^{\mathrm{MMM}}$ with respect to high order element is also demonstrated by employing the 8-node quadratic quadrilateral to solve a beam bending problem considering surface effect. In addition, the introduced sampling error with $\mathrm{SR}^{\mathrm{MMM}}$ that is analogous to numerical integration error with quadrature rule in FEM is very small.
\end{abstract}

Keywords: Surface effect; multiresolution molecular mechanics; multiscale modeling; summation rule; quadrature rule; finite element method 


\section{Introduction}

Nanostructures with decreased size and dimensionality such as nanowires, nanotubes, nanofilms and nanoparticles may exhibit increasingly unusual properties, i.e., optical, mechanical, electrical and thermal, compared to those of the macroscopic ones. These size-dependent material properties have been experimentally viewed in [1-8] and computationally observed in [9-14]. For example, by in-situ tensile testing, Young's modulus, yield strength and ultimate tensile strength have been found to increase as the diameter of silver nanowire decreases [7]. Through largescale atomistic simulations, the tensile yield strength increase and compressive yield strength decrease are observed by decreasing the diameter of metallic glass samples [11] and the softening of nanoporous aluminum are found to significantly reduced by reducing the size of ligaments and joints that connect them in the structure [15].

Nanotechnologies such as nanoscale resonant sensors, micro- and nano-electro-mechanical systems (M/NEMS), and stretchable nano-electronics, can be improved by a better understanding of size-dependent mechanical properties of nanostructures [16, 17]. Applications range from mass and force detection[18-20], frequency synthesis [21, 22] to mechanical switches [23], to name a few.

The key factor in which nanostructures are different from their macroscopic counterpart lies in that the increasing surface-to-volume ratio at nanoscale is significantly larger than that at macroscopic scale. The presence of abundant free surfaces at nanoscale alters the effective material behavior in both the elastic region and beyond. In particular, surface relaxation changes the local atomic configuration close to the surface due to the lack of bonding neighbors, which in turn alter the atomic interactions and thus affect the effective elastic moduli. Since the percentage of surface atoms increases with increasing surface-to-volume ratio that characterizes nanomaterials, the surface effects become significant with decreasing material dimensionality.

The challenge in modelling surface effects for nano-sized structures is that, on the one hand, classical continuum theory that represents bulk material behavior cannot be applied directly but have to be modified to incorporate surface effects; on the other hand, traditional molecular mechanics is limited by the model size due to the demanding computational costs. A wide range of existing approaches have been proposed to solve this issue. In general, existing models may be classified into three groups: (I) enhanced classical continuum mechanics [9, 24-38], (II) atomic-based continuum analysis [39-43] and (III) coarse-grained atomistic approaches [44-48].

Some of the works based on enhanced classical continuum model depend on further development of the surface elasticity formulation proposed by Gurtin and Murdoch [49]. In the theory of surface elasticity, a surface is normally considered as a two-dimensionally (2D) heterogeneous thin film bonded perfectly to the bulk material such that their displacements are continuous across the interface. The general idea is to introduce a surface stress tensor to augment the bulk stress tensor typically employed in continuum mechanics. As such, the conventional boundary conditions need to be modified to account for the presence of surface stresses. One major shortcoming of these models is that surface stress components perpendicular 
to or out-of-surface plane may not be able to be captured due to the equilibrium required between bulk and surface [50].

This has motivated the development of atomic-based continuum analysis. The well-known surface Cauchy-Born (SCB) theory proposed by Park in [39-41, 50, 51] is a representative example in this category. The boundary Cauchy-Born model is similar to SCB except that it also considers corner and edge effects. The main difference of models in group II from approaches in category I lies in that the derivation of the constitutive description in group II is from an underlying atomic structure and interatomic potential, rather than empirical rules and phenomenological models as employed in group I. The key feature of SCB to capture surface effects is based on decomposing the potential energy into bulk and surface components to naturally form a variational framework work that can be directly incorporated into standard nonlinear finite element analysis. Recently thermal effect has been incorporated into SCB based on employing temperature-dependent interatomic potentials [52]. However, SCB cannot be directly applied to model surface-defect interaction because SCB is inherently based on the continuum framework and hence is somewhat limited.

This aforementioned drawback calls for a powerful multiscale model that may not depend on the continuum framework such as the constitutive stress-strain relationship but pure atomistic description, which motivated the development of coarse-grained atomistic models [45-48, 53-59] in group III. The nonlocal quasicontinuum (QC) method [45-47, 53-56, 60] is a typical representative method and has been recently applied to study surface effect in nanoscale structures based on the newly proposed summation rules in [45]. Since the proposed summation rules employed in the quasicontinuum framework are specifically designed for linear interpolation shape functions, the consistent extension to high order interpolation shape functions is not clear, as has been clearly observed in [61, 62]. Since linear shape function is not well suited for deformations such as bending and bending experiments are widely employed to identify size-dependent mechanical properties of nanostructures, there is a need to develop a coarse-grained atomistic framework that is universally applicable to general types of interpolation shape functions and deformations. A more detailed discussion on models regarding size-dependent mechanical properties can be found in several review papers [63-65].

In the present work, the recently proposed multiresolution molecular mechanics (MMM) is applied to study surface effects of nanostructures. In a general sense, MMM can be thought as a generalized nonlocal quasicontinuum approach that provides a unified mathematical framework from linear to any high-order interpolation shape functions in [57], where an optimal summation rule $\mathrm{SR}^{\mathrm{MMM}}$ is proposed. $\mathrm{SR}^{\mathrm{MMM}}$ consists of the bulk summation rule $\mathrm{SR}^{\mathrm{B}}$ and the surface summation rule $\mathrm{SR}^{\mathrm{S}}$. $\mathrm{SR}^{\mathrm{B}}$ has been shown to demonstrate better performance, compared to many other summation rules such as Gauss-quadrature-like rule through a relatively comprehensive numerical studies for bilinear and quadratic shape functions [57]. The adaptive analysis, implementation and efficiency of MMM with respect to linear element has been recently reported in $[66,67]$. The focus of the present work is primarily on the performance of the surface summation rule $\mathrm{SR}^{\mathrm{S}}$. As a demonstration, the 4-node bilinear quadrilateral, 8-node quadratic quadrilateral and 8-node hexahedral elements are employed with $\mathrm{SR}^{\mathrm{S}}$ in $2 \mathrm{D}$ and $3 \mathrm{D}$, respectively, 
to solve surface relaxation and beam bending problems. The uniqueness of the present approach is the universal applicability to interpolation shape functions and different deformation types. Though spatial and temporal coarse-graining are equally important, the present work focuses on coarse-graining in space for statics. Techniques employed to coarse-grain temporal scale at finite temperatures [68-71] may be applied to the present work.

The remainder of this paper is organized as follows. Section 2 reviews the methodology of $\mathrm{MMM}$ and illustrates how $\mathrm{SR}^{\mathrm{S}}$ and $\mathrm{SR}^{\mathrm{B}}$ are derived and can be consistently incorporated into $\mathrm{SR}^{\mathrm{MMM}}$. Section 3 defines error norms and specifically constructed error models to identify different error sources quantitatively. Section 4 presents several numerical examples to demonstrate the benefits of the proposed approach, and Section 5 concludes with closing remarks on the performance of $\mathrm{SR}^{\mathrm{MMM}}$ to capture surface effects.

\section{Methodology}

\subsection{Overview}

In this section, the general idea of MMM is reviewed by analogy to the well-known finite element method (FEM) in conventional continuum mechanics. In Fig. 1a, a finite element with shape function $\phi$ is introduced to approximate the original continuum model and the potential energy $E_{c}$ can be approximated as:

$$
E_{c}=\int_{\Omega^{c}} W d \Omega^{c} \cong \tilde{E}_{c}=\sum_{i \in \mathcal{N}_{Q}} w_{i}^{c} W_{i}
$$

where $W$ is potential energy density, $\Omega^{c}$ denotes the continuum domain, $\mathcal{N}_{Q}$ represents the index set of $N_{Q}$ quadrature points and $w_{i}^{c}$ is the associated weight for a quadrature point $i \in \mathcal{N}_{Q}$. In the present work, calligraphic letter $\mathcal{N}$ represents index set and the corresponding Roman letter $N$ defines its cardinality.

In general, Gauss quadrature is widely employed to evaluate the energy integral in Eq. (1) and there is a standard way to determine the optimal number of quadrature points needed and the associated position and weight for each quadrature point. Similarly, in Fig. 1b, a finite element mesh is also employed to reduce the degrees of freedom of the original atomistic model and the potential energy $E_{a}$ is approximated as:

$$
E_{a}=\sum_{i \in \mathcal{N}_{A}} E_{i} \cong \tilde{E}_{a}=\sum_{i \in \mathcal{N}_{S}} w_{i}^{a} E_{i}
$$

where $\mathcal{N}_{A}$ is the index set of the $N_{\mathrm{A}}$ atoms in the original atomistic model, $E_{i}$ is the atomic site energy of an atom $i \in \mathcal{N}_{A}, \mathcal{N}_{S}$ denotes the index set of the chosen $N_{\mathrm{S}}$ sampling or quadrature-type atoms and $w_{i}^{a}$ is the associated weight for a sampling atom $i \in \mathcal{N}_{S}$. The red dots in Fig. 1 are either the conventional finite element nodes for continuum mechanics or the rep-atoms or nodal atoms for atomistic modeling. And the blue atoms represent either a quadrature point to evaluate an integral or a sampling atom to calculate a finite summation. Also, the gray atoms in Fig. 1b are called non-sampling atoms (NSAs). The reason is that these atoms are not the degrees of 
freedom (DOFs) of the coarse-grained system and their energy are not considered explicitly but sampled by the energy of blue dots (sampling atoms).

As conventional finite element nodes and rep-atoms play similar roles in their respective modeling method, we do not differentiate the coloring approach between coarse-grained continuum and atomistic models, so is the same for quadrature and quadrature-type dots. This coloring scheme will be consistently employed in this work unless otherwise stated. We note that if a quadrature point coincides with a finite element node, as it may occur for high order elements, we will clearly point that out. In addition, any symbol with a subscript or superscript " $c$ " employed for continuum model has the same physical meaning as the same symbol with a subscript or superscript " $a$ " utilized for the atomistic system.

The key issue in Eq. (2) is in developing a standard and systematic theory to determine the optimal number $N_{\mathrm{S}}$, weight $w_{i}^{a}$ and position for each sampling atom for general finite element shape functions, which is analogous to the Gauss quadrature employed in conventional FEM. A previous work by the authors in [57] has addressed this issue by deriving the MMM summation rule $\mathrm{SR}^{\mathrm{MMM}}$, which will be briefly reviewed in the following section.

\subsection{MMM summation rule $\mathbf{S R}^{\mathrm{MMM}}$}

In conventional FEM, it is well-known that the order of employed quadrature rule is shapefunction dependent [72]. In the following derivation, we will show that the order of employed $\mathrm{SR}^{\mathrm{MMM}}$ also depends on the shape function employed. This is different from the summation rule proposed in [56] which claims that the calculation of weight $w_{i}^{a}$ is independent of the interpolation scheme employed.

For simplicity, external loads are not considered and linear elasticity model is assumed for continuum mechanics. Then the potential energy approximation $\tilde{E}_{c}$ in Eq. (1) can be expressed in the form of Eq. (3) as:

$$
\tilde{E}_{c}=\frac{1}{2} \sum_{i \in \mathcal{N}_{n}^{c}} \sum_{j \in \mathcal{N}_{n}^{c}} \mathbf{u}_{i}^{c T} \mathrm{~K}_{i j}^{c} \mathbf{u}_{j}^{c}
$$

where $\mathcal{N}_{n}^{c}$ is the index set of $N_{n}^{c}$ finite element nodes, $\mathbf{u}_{i}^{c}$ is the nodal displacement vector for a node $i \in \mathcal{N}_{n}^{c}$ and $\mathrm{K}_{i j}^{c}$ represents the nodal stiffness matrix for nodes $i$ and $j . \mathrm{K}_{i j}^{c}$ can be formulated as:

$$
\mathrm{K}_{i j}^{c}=\int_{\Omega^{c}} \mathrm{~B}_{i}^{c^{T}} \mathrm{D}^{c} \mathrm{~B}_{j}^{c} d \Omega^{c}
$$

where $\mathrm{D}^{c}$ is a matrix of material constants that describes the continuum constitutive behavior, $\mathrm{B}_{i}^{c}$ is the strain matrix for node $i$ and can be defined in three dimensional (3D) setting as: 


$$
\mathrm{B}_{i}^{c}=\left[\begin{array}{ccc}
\phi_{i, x} & 0 & 0 \\
0 & \phi_{i, y} & 0 \\
0 & 0 & \phi_{i, z} \\
\phi_{i, y} & \phi_{i, x} & 0 \\
0 & \phi_{i, z} & \phi_{i, y} \\
\phi_{i, z} & 0 & \phi_{i, x}
\end{array}\right]
$$

From Eq. (5), we note that the order of quadrature rule needed to evaluate $\widetilde{E}_{c}$ or $K_{i j}^{C}$ depends on the order of the product of the two shape function derivatives. As an example, let $\Pi_{i j}^{c}$ denote the product of shape function derivatives in one dimension (1D), as follows:

$$
\Pi_{i j}^{c}=\phi_{i, x} \phi_{j, x}
$$

From Eqs. (3)-(6), we point out that the distribution of $\widetilde{E}_{c}$ is determined by the order of function bases in $\Pi_{i j}^{a}$. For example, we let $\phi$ be the 1D quadratic shape function. Then, by a simple calculation, one can see that the solid line in Fig. 2a describing the distribution of $\tilde{E}_{c}$, i.e. the energy density of the FEM model can be defined as:

$$
\mathrm{f}^{c}\left(x_{c}\right)=b_{c}^{0}+b_{c}^{1} x_{c}+b_{c}^{2} x_{c}^{2}
$$

where $b_{c}^{i}, i=0-2$ are constants dependent on the nodal displacement vector $\mathbf{u}_{i}^{c}, i \in \mathcal{N}_{n}^{c}$ and material constants in matrix $\mathrm{D}^{\mathrm{c}}, x_{c}$ is a material point position that continuously spans the continuum domain $\Omega^{c}$. Then $\tilde{E}_{c}$ can be evaluated as:

$$
\tilde{E}_{c}=\int_{\Omega^{c}} \mathrm{f}^{c}\left(x_{c}\right) \mathrm{d} \Omega^{c}
$$

Since $\mathrm{f}^{c}\left(x_{c}\right)$ is a continuous quadratic function, then the optimal number of quadrature points $N_{Q}$ is 2 and their corresponding weight $w_{i}^{c}$ in Eq. (1) can be determined by Gauss quadrature rule, which shows how the employed shape function derivatives determine the order of selected quadrature rule in conventional FEM. Next, we will show how $\mathrm{SR}^{\mathrm{MMM}}$ is derived and related to the employed shape function differences.

Similarly, for an atomistic model, a nonlocal linear spring potential is assumed for the interatomic interaction to facilitate demonstration of $\mathrm{SR}^{\mathrm{MMM}}$. As such, the potential energy approximation $\widetilde{E}_{a}$ in Eq. (2) can be expressed as:

$$
\widetilde{E}_{a}=\frac{1}{2} \sum_{i \in \mathcal{N}_{n}^{a}} \sum_{j \in \mathcal{N}_{n}^{a}} \mathbf{u}_{i}^{a T} K_{i j}^{a} \mathbf{u}_{j}^{a}
$$

where $\mathrm{K}_{i j}^{a}$ is defined as:

$$
\begin{gathered}
\mathrm{K}_{i j}^{a}=\frac{1}{2} \sum_{\alpha \in \mathcal{N}_{A}} \sum_{\beta \in \mathcal{N}_{\alpha}} \mathrm{B}_{i}^{a^{T}} \mathrm{D}^{a} \mathrm{~B}_{j}^{a} \\
\mathrm{~B}_{i}^{a}=\left[\phi_{i}\left(\mathbf{r}_{\alpha 0}\right)-\phi_{i}\left(\mathbf{r}_{\beta 0}\right)\right] \mathbf{I}
\end{gathered}
$$

where $\mathcal{N}_{\alpha}$ is the index set of $N_{\alpha}$ atoms that interacts with atom $\alpha \in \mathcal{N}_{A}, \mathbf{r}_{\alpha 0}$ denotes the initial position vector of atom $\alpha$ and $\mathrm{D}^{a}$ is the material constants or the local stiffness matrix that 
describes the interatomic interaction in each direction. For linear spring potentials in $3 \mathrm{D}$, $\mathbf{I}$ is the 3 by 3 identity matrix. Note that $\mathrm{B}_{i}^{a}$ is different from $\mathrm{B}_{i}^{c}$ since there is no shape function directive involved and we do not have shear strain in the interatomic potential. Physically, this difference is due to the fact that the constitutive relationship in continuum mechanics generally contains six strains; however, the interatomic potential in atomistic modeling depends on pair distance difference in $x, y$ and $z$ directions.

For lattice or crystal structures, the neighboring position vector $\mathbf{r}_{\beta 0}$ can be determined in terms of $\mathbf{r}_{\alpha 0}$, as follows:

$$
\mathbf{r}_{\beta 0}=\mathbf{r}_{\alpha 0}+\mathbf{C}\left(a_{0}\right)
$$

where $\mathbf{C}$ is a non-zero constant vector in terms of the lattice constant $a_{0}$. If Eq. (12) is plugged back into Eq. (11), we note that the evaluation of $\mathrm{K}_{i j}^{a}$ is determined by the product of the shape function difference calculated at an atom $\alpha \in \mathcal{N}_{A}$. For example, let $\phi$ be the one-dimensional quadratic shape function. Then $\mathbf{I}$ is 1 and $\mathrm{D}^{a}$ is the pair-wise spring constant. Let $\Pi_{i j}^{a}$ denote the shape function difference product as follows:

$$
\Pi_{i j}^{a}=\left[\phi_{i}\left(\mathbf{r}_{\alpha 0}\right)-\phi_{i}\left(\mathbf{r}_{\alpha 0}+\mathbf{C}\left(a_{0}\right)\right)\right]\left[\phi_{j}\left(\mathbf{r}_{\alpha 0}\right)-\phi_{j}\left(\mathbf{r}_{\alpha 0}+\mathbf{C}\left(a_{0}\right)\right)\right]
$$

By a simple calculation (See Section 2.4 or Appendix A in [57] for detail), one can see that $\Pi_{i j}^{a}$ is also quadratic in terms of the components of $\mathbf{r}_{\alpha 0}$. Then, from Eqs. (9)-(13), we note that the dashed line in Fig. $2 b$ describing the distribution of $\tilde{E}_{a}$, i.e. the atom-wise energy can also be defined as:

$$
\mathrm{f}^{a}\left(x_{a}\right)=b_{a}^{0}+b_{a}^{1} x_{a}+b_{a}^{2} x_{a}^{2}
$$

where $b_{a}^{i}, i=0-2$ are constants in terms of nodal displacement vector $\mathbf{u}_{i}^{a}, i \in \mathcal{N}_{n}^{a}$ and material constants in the employed linear spring potential, $x_{a}$ is an atomic position that discretely spans atomic domain $\Omega^{a}$. Then $\tilde{E}_{a}$ can be evaluated as:

$$
\tilde{E}_{a}=\sum_{i \in \mathcal{N}_{A}} \mathrm{f}^{a}\left(x_{a}^{i}\right)
$$

where $x_{a}^{i}$ is the atomic position of atom $i \in \mathcal{N}_{A}$.

We note that the difference between the energy of a surface atom and that of a bulk atom is not distinguished so far for the sake of introducing the general idea of $\mathrm{SR}^{\mathrm{MMM}}$. From Fig. 2, one can see that the similarity between $\tilde{E}_{c}$ and $\tilde{E}_{a}$ lies in that they have the same distribution order. The main difference between them is that $\mathrm{f}^{c}\left(x_{c}\right)$ is a continuous function (solid line) in terms of $x_{c}$ but $\mathrm{f}^{a}\left(x_{a}\right)$ is a set of discrete points in terms of $x_{a}$ that pass through the dashed line. As such, three sampling (or quadrature-type) atoms are required to exactly represent the dashed energy distribution line. Then the optimal number for sampling atoms $N_{\mathrm{S}}$ is 3 and the corresponding weight $w_{i}^{a}$ in Eq. (2) can be determined as in any curve-fitting process. For example, as the dashed line in Fig. $2 b$ has a quadratic form, three sampling atoms ( 2 blue dots and the interior red node) are selected. Then the dashed line can be expressed as: 


$$
\mathrm{f}^{a}\left(x_{a}\right)=\sum_{i \in \mathcal{N}_{S}} \Phi_{i}\left(x_{a}\right) E_{i}
$$

where $\Phi_{i}$ is defined as:

$$
\Phi_{i}=\prod_{i \neq j, i \in \mathcal{N}_{S}, j \in \mathcal{N}_{S}} \frac{x_{a}-x_{a}^{j}}{x_{a}^{i}-x_{a}^{j}}
$$

As can be seen from Eq. (17), $\Phi_{i}$ satisfies the partition of unity and Kronecker delta property, as follows:

$$
\begin{gathered}
\sum_{i \in \mathcal{N}_{S}} \Phi_{i}\left(x_{a}^{k}\right)=1, \forall k \in \mathcal{N}_{A} \\
\Phi_{i}\left(x_{a}^{j}\right)=\delta_{i j}, \forall i, j \in \mathcal{N}_{S}
\end{gathered}
$$

Then the energy summation $\widetilde{E}_{a}$ at the discrete atoms is calculated as:

$$
\tilde{E}_{a}=\sum_{j \in \mathcal{N}_{A}} \mathrm{f}\left(x_{a}^{j}\right)=\sum_{j \in \mathcal{N}_{A}} \sum_{i \in \mathcal{N}_{S}} \Phi_{i}\left(x_{a}^{j}\right) E_{i}=\sum_{i \in \mathcal{N}_{S}} \sum_{j \in \mathcal{N}_{A}} \Phi_{i}\left(x_{a}^{j}\right) E_{i}
$$

Comparing Eqs. (20) and (2), we note that:

$$
w_{i}^{a}=\sum_{j \in \mathcal{N}_{A}} \Phi_{i}\left(x_{a}^{j}\right)
$$

The above curve-fitting-like process to determine the optimal number of sampling atoms $N_{\mathrm{S}}$ and the corresponding weight $w_{i}^{a}$ forms the MMM summation rule $\mathrm{SR}^{\mathrm{MMM}}$, which clearly shows how $\mathrm{SR}^{\mathrm{MMM}}$ is related to the employed shape function differences. For more details about deriving $\mathrm{SR}^{\mathrm{MMM}}$, we refer the interested reader to our previous work in [57].

It is worth to note that the above MMM analogy to FEM is introduced from an energy point of view. Though FEM is equivalent to an energy minimization problem under certain circumstances, FEM is, in general, based on the weak form of the original ordinary or partial differential equations, and on weighting the residual integral to zero by setting the trial function space also as the test function space. On the other hand, molecular mechanics lies in the energy minimization for statics considered here. The aforementioned analogy is only employed to better the understanding of the introduced MMM framework.

\subsection{SR ${ }^{\mathrm{MMM}}$ decomposition: $\mathrm{SR}^{\mathrm{B}}$ and $\mathrm{SR}^{\mathrm{S}}$}

In this section, we will explain why there is a need to distinguish the difference between the energy distribution of surface atoms and that of bulk atoms and hence decompose $\mathrm{SR}^{\mathrm{MMM}}$ into the bulk summation rule $\mathrm{SR}^{\mathrm{B}}$ and the surface summation rule $\mathrm{SR}^{\mathrm{S}}$.

We note that $\mathcal{N}_{\alpha}$ in Eq. (10) is the index set of $N_{\alpha}$ neighboring atoms that interact with an atom $\alpha \in \mathcal{N}_{A}$. Physically, the fundamental difference between surface atoms and bulk counterpart is that surface atoms are not fully coordinated and lack neighboring atoms. As such, $N_{\alpha}^{S}$, the number of neighboring atoms of a surface atom $\alpha$ that belongs to the index set of surface atoms $\mathcal{N}_{A}^{S}$, is different from $N_{\alpha}^{B}$, the number of neighboring atoms that interacts with a bulk atom $\alpha$ that resides in the index set of bulk atoms $\mathcal{N}_{A}^{B}$. As a result, the energy distribution of surface atoms, $\mathrm{f}_{S}^{a}\left(x_{a}\right)$, will be different from that of bulk atoms, $\mathrm{f}_{B}^{a}\left(x_{a}\right)$, even though they both reserve the 
quadratic form in Eq. (14) for the one-dimensional quadratic shape function considered in Section 2.2. Mathematically, this is because the coefficient $b_{a}^{i}, i=0-2$, in Eq. (14) take different values for $\mathrm{f}_{S}^{a}\left(x_{a}\right)$ and $\mathrm{f}_{B}^{a}\left(x_{a}\right)$, respectively. This is schematically demonstrated in Fig. 3, where the energy of surface atoms (empty circles in rectangle boxes) does not follow the bulk energy distribution curve (dashed line).

For the reasons discussed above, $\mathrm{SR}^{\mathrm{MMM}}$ is decomposed into $\mathrm{SR}^{\mathrm{B}}$ and $\mathrm{SR}^{\mathrm{S}}$. Basically, $\mathrm{SR}^{\mathrm{B}}$ is employed to treat bulk atoms and $\mathrm{SR}^{\mathrm{S}}$ is used to treat surface atoms to accurately represent the energy distribution over the whole atomic domain $\Omega^{a}$. The methodology to determine the optimal number of sampling atom $N_{\mathrm{S}}$ and their respective weights $w_{i}^{a}$ in $\mathrm{SR}^{\mathrm{B}}$ and $\mathrm{SR}^{\mathrm{S}}$ are exactly the same as for $\mathrm{SR}^{\mathrm{MMM}}$ in Section 2.2.

We note that the performance of $\mathrm{SR}^{\mathrm{B}}$ has been studied by a relatively comprehensive numerical examples in [57], where $\mathrm{SR}^{\mathrm{B}}$ is demonstrated to outperform Gauss-quadrature-like rule and some other summation rules. As such, $\mathrm{SR}^{\mathrm{B}}$ will be directly employed to treat the bulk atomistic region in this work. The focus of the present research will be regarding the performance of $\mathrm{SR}^{\mathrm{S}}$ to capture surface effects.

\subsection{Design of employed $S^{S}$}

In this section, the employed $\mathrm{SR}^{\mathrm{S}}$ in $2 \mathrm{D}$ and $3 \mathrm{D}$ will be introduced for the respective 4-node bilinear quadrilateral, 8-node quadratic quadrilateral and 8-node hexahedral elements.

The derivation of $\mathrm{f}^{a}\left(x_{a}\right)$ in Eq. (14) for the 4-node quadrilateral element in 2D is given here for demonstration purpose. As such, the shape function $\phi_{i}$ for a node $i \in \mathcal{N}_{n}^{a}$ takes the following form:

$$
\phi_{i}(x, y)=a_{i}^{0}+a_{i}^{1} x+a_{i}^{2} y+a_{i}^{3} x y
$$

where $x$ and $y$ are the respective projection of position vector $\mathbf{r}=\left(\begin{array}{l}x \\ y\end{array}\right)$ of an arbitrary atom in $x$ and $y$ directions, $a_{i}^{\alpha}, \alpha=0-3$ are constants in terms of the chosen nodal positions to construct $\phi_{i}$. Let $\mathrm{C}_{x}$ and $\mathrm{C}_{y}$ be the projection of the non-zero constant vector $\mathbf{C}=\left(\begin{array}{l}\mathrm{C}_{x} \\ \mathrm{C}_{y}\end{array}\right)$ in Eq. (12) in $x$ and $y$ directions, respectively. Then $\phi_{i}(\mathbf{r}+\mathbf{C})$ in Eq. (13) is calculated as:

$$
\phi_{i}\left(x+\mathrm{C}_{x}, y+\mathrm{C}_{y}\right)=a_{i}^{0}+a_{i}^{1}\left(x+\mathrm{C}_{x}\right)+a_{i}^{2}\left(y+\mathrm{C}_{y}\right)+a_{i}^{3}\left(x+\mathrm{C}_{x}\right)\left(y+\mathrm{C}_{y}\right)
$$

such that the shape function difference is expressed as:

$$
\phi_{i}(x, y)-\phi_{i}\left(x+\mathrm{C}_{x}, y+\mathrm{C}_{y}\right)=b_{i}^{0}+b_{i}^{1} x+b_{i}^{2} y
$$

where $b_{i}^{\alpha}, \alpha=0-2$ are given in terms of $a_{i}^{\alpha}, \alpha=0-3$ and constants $\mathrm{C}_{x}$ and $\mathrm{C}_{y}$. Then, $\Pi_{i j}^{a}$ in Eq. (13) is defined as:

$$
\Pi_{i j}^{a}=c_{i j}^{0}+c_{i j}^{1} x+c_{i j}^{2} y+c_{i j}^{3} x y+c_{i j}^{4} x^{2}+c_{i j}^{5} y^{2}
$$


where $c_{i j}^{\alpha}, \alpha=0-5$, are constants given in terms of $b_{i}^{\alpha}$ and $b_{j}^{\alpha}, \alpha=0-2$. Finally, $\mathrm{f}^{a}\left(x_{a}\right)$ in Eq. (14) takes the following form:

$$
\mathrm{f}^{a}\left(x_{a}\right)=b_{a}^{0}+b_{a}^{1} x+b_{a}^{2} y+b_{a}^{3} x y+b_{a}^{4} x^{2}+b_{a}^{5} y^{2}
$$

where $b_{a}^{i}, i=0-5$ are constants in terms of the nodal displacement vector $\mathbf{u}_{i}^{a}, i \in \mathcal{N}_{n}^{a}$ and material constants in the employed linear spring potential and the coefficients in the given bilinear shape function.

From Eq. (26), we note that the optimal number of sampling atoms $N_{S}^{B}$ for $\mathrm{SR}^{\mathrm{B}}$ is 6 and $\Phi_{i}$ in Eq. (17) also has a fully quadratic form and can be determined by a curve-fitting-like process, as discussed in Section 2.2, such that $w_{i}^{a}$ is also identified correspondingly in Eq. (21).

For the employed 8-node quadratic quadrilateral element in $2 \mathrm{D}, \mathrm{f}^{a}\left(x_{a}\right)$ can be derived following the same process as for the 4-node quadrilateral element. It is found that $\mathrm{f}^{a}\left(x_{a}\right)$ has a full cubic order plus some fourth order terms, as shown in Eq. (27):

$$
\begin{aligned}
& \mathrm{f}^{a}\left(x_{a}\right)=b_{a}^{0}+b_{a}^{1} x+b_{a}^{2} y+b_{a}^{3} x y+b_{a}^{4} x^{2}+b_{a}^{5} y^{2}+b_{a}^{6} x^{3}+b_{a}^{7} x^{2} y+b_{a}^{8} x y^{2}+b_{a}^{9} y^{3}+ \\
& b_{a}^{10} x^{4}+b_{a}^{11} x^{2} y^{2}+b_{a}^{12} y^{4}
\end{aligned}
$$

In this work, we will discard the uncompleted fourth order terms and assume $f^{a}\left(x_{a}\right)$ takes the complete third order form:

$$
\mathrm{f}^{a}\left(x_{a}\right)=b_{a}^{0}+b_{a}^{1} x+b_{a}^{2} y+b_{a}^{3} x y+b_{a}^{4} x^{2}+b_{a}^{5} y^{2}+b_{a}^{6} x^{3}+b_{a}^{7} x^{2} y+b_{a}^{8} x y^{2}+b_{a}^{9} y^{3}
$$

As such, for the $2 \mathrm{D}$ quadratic quadrilateral element, $N_{S}^{B}$ for $\mathrm{SR}^{\mathrm{B}}$ is $10, \Phi_{i}$ in Eq. (17) also has a fully cubic form and $w_{i}^{a}$ can be determined as in Eq. (21).

Similarly, for the employed 8-node hexahedral element in 3D, we find that $\mathrm{f}^{a}\left(x_{a}\right)$ possesses a full quadratic order plus some mixed high order terms, as shown in Eq. (29):

$\mathrm{f}^{a}\left(x_{a}\right)=b_{a}^{0}+b_{a}^{1} x+b_{a}^{2} y+b_{a}^{3} z+b_{a}^{4} x y+b_{a}^{5} x z+b_{a}^{6} y z+b_{a}^{7} x^{2}+b_{a}^{8} y^{2}+b_{a}^{9} z^{2}+b_{a}^{10} x^{2} y+$ $b_{a}^{11} x^{2} z+b_{a}^{12} y^{2} x+b_{a}^{13} y^{2} z+b_{a}^{14} z^{2} x+b_{a}^{15} z^{2} y+b_{a}^{16} x^{2} y^{2}+b_{a}^{17} x^{2} z^{2}++b_{a}^{18} y^{2} z^{2}+$ $b_{a}^{19} x^{2} y z+b_{a}^{20} y^{2} x z+b_{a}^{21} z^{2} x y$

Again, we will discard the mixed terms and assume $\mathrm{f}^{a}\left(x_{a}\right)$ takes the fully quadratic form:

$$
\mathrm{f}^{a}\left(x_{a}\right)=b_{a}^{0}+b_{a}^{1} x+b_{a}^{2} y+b_{a}^{3} z+b_{a}^{4} x y+b_{a}^{5} x z+b_{a}^{6} y z+b_{a}^{7} x^{2}+b_{a}^{8} y^{2}+b_{a}^{9} z^{2}
$$

such that $N_{S}^{B}$ is 10 and $\Phi_{i}$ and $w_{i}^{a}$ can be determined correspondingly, following the above discussed curve-fitting-like process.

So far, the optimal number of sampling atoms $N_{S}^{B}$ and their corresponding weight $w_{i}^{a}$ in bulk summation rule $\mathrm{SR}^{\mathrm{B}}$ are discussed and determined. Next, we will show how $N_{S}^{S}$, the optimal number of sampling atoms in $\mathrm{SR}^{\mathrm{S}}$, and their corresponding weight $w_{i}^{a}$ are identified.

For the rectangle triangular lattice in 2D, as shown in Fig. 4, we note that either $x$ or $y$ is constant for the respective surface edge and non-bulk edges (dashed lines). As such, $\mathrm{f}^{a}\left(x_{a}\right)$ in Eq. (26) 
can be reduced to fewer terms. For instance, for the surface edge on the left hand side, $y$ is constant such that, $\mathrm{f}_{S}^{a}\left(x_{a}\right)$ can be reduced to:

$$
\mathrm{f}_{s}^{a}\left(x_{a}\right)=\varepsilon_{a}^{0}+\varepsilon_{a}^{1} x+\varepsilon_{a}^{2} x^{2}
$$

where $\varepsilon_{a}^{i}, i=0-2$ are constants in terms of $b_{a}^{i}$ in Eq. (26) and $y$. Then from Eq. (31), it is clearly seen that $N_{S}^{S}$ is 3 and $w_{i}^{a}$ in $\mathrm{SR}^{\mathrm{S}}$ can be calculated as in $\mathrm{SR}^{\mathrm{B}}$. If a non-bulk layer is considered (Fig. $4 c$ and $4 d$ ), the same $\mathrm{SR}^{\mathrm{S}}$ can also be applied, as in the $2 \mathrm{D}$ example in Section 4 . Figure 4 schematically demonstrates the idea on how $\mathrm{SR}^{\mathrm{MMM}}\left(\mathrm{SR}^{\mathrm{S}}+\mathrm{SR}^{\mathrm{B}}\right)$ is employed for the four-node bilinear quadrilateral element with (Fig. 4a and Fig. 4c) and without a corner atom (Fig. 4b and Fig. 4d).

For the 8-node quadratic quadrilateral element, the same analysis can be directly applied to such that $\mathrm{f}^{a}\left(x_{a}\right)$ in Eq. (32) is reduced as:

$$
\mathrm{f}_{s}^{a}\left(x_{a}\right)=\varepsilon_{a}^{0}+\varepsilon_{a}^{1} x+\varepsilon_{a}^{2} x^{2}+\varepsilon_{a}^{3} x^{3}
$$

Then $N_{S}^{S}$ is 4 (as shown in Fig. 9b) and $w_{i}^{a}$ in $\mathrm{SR}^{\mathrm{S}}$ can be determined accordingly.

In order to distinguish the aforementioned sampling atoms from secondary sampling atoms (SSAs) that will be introduced later in this section, we name these sampling atoms primary sampling atoms (PSAs). In addition, the PSAs where $\mathrm{SR}^{\mathrm{S}}$ is applied are named surface PSAs (SPSAs) and PSAs where $\mathrm{SR}^{\mathrm{B}}$ is applied are called bulk PSAs (BPSAs).

The same idea can be applied to a face-centered cubic (FCC) lattice in 3D. For each of the surface and non-bulk layers, either $x, y$ or $z$ is constant such that $\mathrm{f}^{a}\left(x_{a}\right)$ in Eq. (30) can also be trimmed to fewer terms. For example, for the surface layer on the bottom, $z$ is a constant such that $\mathrm{f}_{S}^{a}\left(x_{a}\right)$ can be redefined as:

$$
\mathrm{f}_{S}^{a}\left(x_{a}\right)=b_{a}^{0}+b_{a}^{1} x+b_{a}^{2} y+b_{a}^{3} x y+b_{a}^{4} x^{2}+b_{a}^{5} y^{2}
$$

where the summation rule $\mathrm{SR}^{\mathrm{MMM}}$ in $2 \mathrm{D}$ can be directly applied such that $N_{S}^{S}$ and $w_{i}^{a}$ can be determined accordingly. We note that, mathematically, $\mathrm{SR}^{\mathrm{S}}$ in $3 \mathrm{D}$ is, in fact, the $\mathrm{SR}^{\mathrm{MMM}}\left(\mathrm{SR}^{\mathrm{B}}+\right.$ $\left.\mathrm{SR}^{\mathrm{S}}\right)$ in $2 \mathrm{D}$ and $\mathrm{SR}^{\mathrm{S}}$ in $2 \mathrm{D}$ is actually the $\mathrm{SR}^{\mathrm{MMM}}\left(\mathrm{SR}^{\mathrm{B}}+\mathrm{SR}^{\mathrm{S}}\right)$ in $1 \mathrm{D}$. This is understandable since, physically, it is obvious that the surfaces of $3 \mathrm{D}$ model are $2 \mathrm{D}$ and the edges of $2 \mathrm{D}$ are $1 \mathrm{D}$. Note that the 2D surface or 1D edge could be curved. In the present work, we focus on regular shapes. Surfaces and edges with curvature will be considered in our future work.

For positions of primary sampling atoms, based on our previous study in [57], we note that different selections of primary sampling atoms in $\mathrm{SR}^{\mathrm{B}}$ (thus called bulk primary sampling atoms) may not produce a significant difference as long as they are selected from the bulk region and are not coplanar. This can be understood from the perspective of the curve-fitting process. For instance, a 2D quadratic surface can be represented by 6 selected points provided that they are not coplanar. The same conclusion applies to the selection of surface sampling atoms in $\mathrm{SR}^{\mathrm{S}}$.

It is worth to note that the energy of corner atoms does not exactly follow $\mathrm{f}_{S}^{a}\left(x_{a}\right)$ or $\mathrm{f}_{B}^{a}\left(x_{a}\right)$ for the reason that the neighbors of corner atoms are located on more surfaces than that of surface or 
bulk atoms. Similarly, the energy of atoms that coincide with $N_{n}^{a}$ finite element nodes (called nodal atoms) and energy of atoms near element edges (called element edge atoms) within a potential cut-off distance $\left(r_{\text {cut }}\right)$ also do not exactly follow $\mathrm{f}_{S}^{a}\left(x_{a}\right)$ or $\mathrm{f}_{B}^{a}\left(x_{a}\right)$ since neighbors of these atoms are located in more finite elements. Based on our previous results in [57], the assumption that the energy of element edge atoms follows $\mathrm{f}_{S}^{a}\left(x_{a}\right)$ or $\mathrm{f}_{B}^{a}\left(x_{a}\right)$ may not introduce significant errors, as will be seen later in Section 4. However, the energy of nodal atoms will be considered explicitly instead of being sampled by energy of primary sampling atoms. For this reason, we call the atoms whose energy are considered explicitly secondary sampling atom (SSA) to represent their own energies (such that the weight $w_{i}^{a}$ is 1 for each $S S A$ ) and we name the aforementioned sampling atoms (blue dots in Figs. 1-4) whose energy is employed to sample the energy of non-sampling atoms (NSA) (gray dots in Figs. 1-4) primary sampling atoms (PSA). A more detailed explanation and atom classification can be found in $[48,57]$.

\subsection{Remarks on Nonlinear Potential}

In conventional FEM, the selected order of quadrature rule to evaluate the energy (Eq. (3)) or stiffness integral (Eq. (4)) is generally determined by the accuracy of Gauss quadrature for linear elasticity. For nonlinear constitutive relationships, a relatively higher order of Gauss quadrature may be employed to improve accuracy. We note that reduced Gauss quadrature may have better performance for problems where internal constraints in the continuum theory being assumed, such as incompressibility or the Kirchhoff transverse shear constraints in bending analysis. Full integration or higher order Gauss quadrature may introduce the so-called shear or volumetric locking issues and thus may deteriorate the accuracy of FEM.

Similarly, $\mathrm{SR}^{\mathrm{MMM}}$ has been introduced by assuming a nonlocal harmonic spring potential, as mentioned earlier. However, since any nonlinear interatomic potential can be well-approximated by a harmonic spring within its convex region, $\mathrm{SR}^{\mathrm{MMM}}$ is expected to be effective for nonlinear potentials, which have been clearly demonstrated in [48, 57-59]. In fact, the proposed $\mathrm{SR}^{\mathrm{MMM}}$ framework is interatomic-potential independent. As such, we do not employ a specific form for the assumed harmonic spring potential in the derivation of $\mathrm{SR}^{\mathrm{MMM}}$. The proposed $\mathrm{SR}^{\mathrm{MMM}}$ can be directly applied to a general form of pair or many-body potential. For cases in which large deformation is expected, a relatively higher order of $\mathrm{SR}^{\mathrm{MMM}}$ may be employed to improve accuracy, as in the case of nonlinear FEM. It is worth to note that whether higher order of $\mathrm{SR}^{\mathrm{MMM}}$ will necessarily guarantee better accuracy or reduced $\mathrm{SR}^{\mathrm{MMM}}$ may have a better performance under certain conditions (for instance, is there "locking-like" problem in MMM? ) is currently under investigation and will be reported later.

Note that for atomistic regions where interatomic potential is within its non-convex domain, full molecular mechanics can be employed since defects are likely to occur in that region. Since MMM is a fully coarse-grained atomistic model and no continuum description is introduced, full molecular mechanics can be directly and organically incorporated into MMM without any modification, as has been shown in [48]. The focus of this work is to propose the $\mathrm{SR}^{\mathrm{S}}$ to complete the $\mathrm{SR}^{\mathrm{MMM}}$ and test its performance. The surface-defect interaction is our ongoing work. 


\subsection{Governing Equations}

In this section, the governing equations of MMM employing $\mathrm{SR}^{\mathrm{MMM}}\left(\mathrm{SR}^{\mathrm{B}}+\mathrm{SR}^{\mathrm{S}}\right)$ will be derived from the potential energy approximation $\widetilde{E}_{a}$ through the variational principle. As mentioned in Section 2.5, we do not assume a specific form of the interatomic potential $E_{i}$ employed below.

Since atoms are classified into PSAs, SSAs and NSAs from the perspective of energy sampling, then $\widetilde{E}_{a}$ in Eq. (2) can be reformed as:

$$
\tilde{E}_{a}=\sum_{i \in \mathcal{N}_{S}} w_{i}^{a} E_{i}=\sum_{i \in \mathcal{N}_{S S A}} E_{i}+\sum_{i \in \mathcal{N}_{P S A}} w_{i}^{a} E_{i}
$$

where $\mathcal{N}_{S S A}$ and $\mathcal{N}_{P S A}$ are the respective index of SSAs and PSAs. Note that $w_{i}^{a}=1$ for each SSA and $w_{i}^{a}$ is determined in $\mathrm{SR}^{\mathrm{MMM}}$ except that Eq. (21) has to be modified as

$$
w_{i}^{a}=\sum_{j \in\left(\mathcal{N}_{A} \backslash \mathcal{N}_{S S A}\right)} \Phi_{i}\left(x_{a}^{j}\right)
$$

to account for the SSAs.

Let $\mathbf{U}^{a}$ denote the displacement vector for all the $N_{n}^{a}$ nodal atoms and $\mathbf{f}_{\alpha}^{\text {ext }}$ be an external force vector applied to an atom $\alpha \in \mathcal{N}_{A}$. Following the variational principle for deriving the equilibrium equations of an MMM model, the negative derivative of $\widetilde{E}_{a}$ with respect to the nodal displacement vector $\mathbf{u}_{i}^{a}, i \in \mathcal{N}_{n}^{a}$, which are the degrees of freedom in MMM as in FEM, must be zero. Then we have:

$$
\mathbf{F}_{i}^{a}\left(\mathbf{U}^{a}\right)=-\sum_{j \in \mathcal{N}_{S S A}} \frac{\partial E_{j}\left(\mathbf{U}^{a}\right)}{\partial \mathbf{u}_{i}^{a}}-\sum_{k \in \mathcal{N}_{P S A}} w_{k}^{a} \frac{\partial E_{k}\left(\mathbf{U}^{a}\right)}{\partial \mathbf{u}_{i}^{a}}+\sum_{\alpha \in \mathcal{N}_{A}} \phi_{i}\left(\mathbf{r}=\mathbf{r}_{\alpha 0}\right) \mathbf{f}_{\alpha}^{e x t}=\mathbf{0}, \forall i \in \mathcal{N}_{n}^{a}
$$

where $\mathbf{f}_{\alpha}^{\text {ext }}$ is partitioned to a nodal atom as in FEM. Equation (36) is the governing equation under the MMM framework for statics. 


\section{Error Quantification}

In this section, different error sources will be defined and identified. In addition, error norms in displacement and energy fields, as inspired by their counterparts for classical FEM, are defined to quantify the performance of MMM.

\subsection{Error sources}

It is well-known that there are two different types of error in conventional FEM: (I) Discretization error that depends on element size and shape function order and (II) numerical integration error that depends on the quadrature rule utilized to evaluate the integrals involved. Similarly, there are also two error types in MMM: (I) Discretization error as in FEM and (II) sampling error that depends on the summation rules employed to evaluate the finite summations involved.

As a simple example, let $\mathbf{U}^{\mathrm{FA}}$ be the displacement solution vector from full atomistic simulation (Model A in Fig. 5a) and $\mathbf{U}^{\mathrm{MMM}}$ be the one from MMM calculation (Model B in Fig. 5b). In addition, in order to quantify the discretization error, a special MMM model (Model C in Fig. 5c) is designed. In Model C, after using finite elements to coarse-grain the full atomistic model, the energy of each atom is considered explicitly such that each atom is taken as a second sampling atom (SSA), denoted as green dots in the present work. We note that the nodal atoms (red dots in Fig. 5) are assigned the atom type SSA. Since they also represent the degrees of freedom, we stay with the color red for them. In Model C, there is only discretization error and no any other error is introduced. As such, Model C is, in general, the most accurate for a given discretization, but it is also the most expensive one. Since there is no primary sampling atom (PSA) in Model C, we denote the displacement field gained from it as $\mathbf{U}_{0 \mathrm{p} * \mathrm{~s}}^{\mathrm{MMM}}$ with "0p" meaning no PSA and "*s" representing SSA everywhere.

As such, for displacement field, the distance between Model A and Model $\mathrm{B}, e_{U}^{\text {tot }}$ can be decomposed as:

$$
\mathbf{U}^{\mathrm{FA}}-\mathbf{U}^{\mathrm{MMM}}=\underbrace{\mathbf{U}^{\mathrm{FA}}-\mathbf{U}_{0 \mathrm{p} * \mathrm{~s}}^{\mathrm{MM}}}_{\text {discretization error }}+\underbrace{\mathbf{U}_{0 \mathrm{p} * \mathrm{~s}}^{\mathrm{MM}}-\mathbf{U}^{\mathrm{MMM}}}_{\text {sampling error }}
$$

where $\mathbf{U}^{\mathrm{FA}}-\mathbf{U}_{0 \mathrm{p} * \mathrm{~s}}^{\mathrm{MMM}}$ is the distance between Model $\mathrm{A}$ and Model $\mathrm{C}$ and is called the discretization error $e_{U}^{\text {disc }} ; \mathbf{U}_{0 \mathrm{M} * \mathrm{~s}}^{\mathrm{MM}}-\mathbf{U}^{\mathrm{MMM}}$ is the distance between Model $\mathrm{C}$ and Model B and is called the sampling error $e_{U}^{s a m}$. The different types of error are schematically shown in Fig. 5

For a given discretization, the discretization error $e_{U}^{\text {disc }}$ is fixed. Then a good indicator to estimate the performance of a coarse-grained model or a summation rule is to identify the sampling error. As many other coarse-grained models do not clearly differentiate and quantify different error sources, we note that the identification of different error types is important, especially when spatial convergence is performed [58].

\subsection{Error norms}


In this section, the two different error sources will be quantified by defining several appropriate error norms.

The following error norms $\left(L_{2}\right.$ and $\left.H_{1}\right)$ are widely used to measure the performance of classical continuum FEM [72]:

$$
\begin{gathered}
e_{U}=\left[\int_{\Omega^{\mathrm{c}}}\left(\mathbf{U}^{\text {exact }}-\mathbf{U}^{\mathrm{FEM}}\right)^{T}\left(\mathbf{U}^{\text {exact }}-\mathbf{U}^{\mathrm{FEM}}\right) \mathrm{d} \Omega^{\mathrm{c}}\right]^{1 / 2} \\
e_{E}=\left[\int_{\Omega^{\mathrm{c}}}\left(\boldsymbol{\varepsilon}^{\text {exact }}-\boldsymbol{\varepsilon}^{\mathrm{FEM}}\right)^{T}\left(\boldsymbol{\varepsilon}^{\text {exact }}-\boldsymbol{\varepsilon}^{\mathrm{FEM}}\right) \mathrm{d} \Omega^{\mathrm{c}}\right]^{1 / 2}
\end{gathered}
$$

where $e_{U}$ and $e_{E}$ are the errors in displacement and energy fields, respectively, $\mathbf{U}^{\text {exact }}$ and $\boldsymbol{\varepsilon}^{\text {exact }}$ are the respective displacement and strain fields from exact solution.

Similarly, the error norms defined in our previous work [58] are taken here to measure the accuracy of MMM in atomistic modeling:

$$
\begin{gathered}
e_{U}^{\text {total }}=\left[\frac{\sum_{i \in \mathcal{N}_{A}}\left(\mathbf{u}_{i}^{\mathrm{FA}}-\mathbf{u}_{i}{ }^{\mathrm{MMM}}\right)^{\mathrm{T}}\left(\mathbf{u}_{i}^{\mathrm{FA}}-\mathbf{u}_{i}{ }^{\mathrm{MMM}}\right)}{\sum_{i \in \mathcal{N}_{A}}\left(\mathbf{u}_{i}^{\mathrm{FA}}\right)^{\mathrm{T}} \mathbf{u}_{i}^{\mathrm{FA}}}\right]^{1 / 2}=\frac{\left\|\mathbf{U}^{\mathrm{FA}}-\mathbf{U}^{\mathrm{MMM}}\right\|_{2}}{\left\|\mathbf{U}^{\mathrm{FA}}\right\|_{2}} \\
e_{E}^{\text {total }}=\left[\frac{\sum_{i \in \mathcal{N}_{A}} \sum_{j \in \mathcal{N}_{i}}\left(\left(\mathbf{r}_{i j}^{\mathrm{FA}}-\mathbf{r}_{i j}^{0}\right)-\left(\mathbf{r}_{i j}^{\mathrm{MMM}}-\mathbf{r}_{i j}^{0}\right)\right)^{\mathrm{T}}\left(\left(\mathbf{r}_{i j}^{\mathrm{FA}}-\mathbf{r}_{i j}^{0}\right)-\left(\mathbf{r}_{i j}^{\mathrm{MMM}}-\mathbf{r}_{i j}^{0}\right)\right)}{\sum_{i \in \mathcal{N}_{A}} \sum_{j \in \mathcal{N}_{i}}\left(\left(\mathbf{r}_{i j}^{\mathrm{FA}}-\mathbf{r}_{i j}^{0}\right)\right)^{\mathrm{T}}\left(\left(\mathbf{r}_{i j}^{\mathrm{FA}}-\mathbf{r}_{i j}^{0}\right)\right)}\right]^{1 / 2}
\end{gathered}
$$

where $\mathbf{u}_{i}^{\mathrm{FA}}$ and $\mathbf{u}_{\boldsymbol{i}}{ }^{\mathrm{MMM}}$ are the displacement vectors from full atomistic calculation and a MMM model, respectively, for an atom $i \in \mathcal{N}_{A} ; \mathcal{N}_{i}$ is the index set of neighbors of the $i$ th atom, $\mathbf{r}_{i j}^{\mathrm{FA}}$ and $\mathbf{r}_{i j}^{\mathrm{MMM}}$ denote the pair distance vector from each model and $\mathbf{r}_{i j}^{0}$ represents the initial pair distance vector. Note that all the errors are normalized by the solution from full atomistic model.

In linear elasticity, the potential energy of a continuum model is a function of the strain field $\boldsymbol{\varepsilon}$, and hence it makes sense that Eq. (39) defines error in energy field. For an atomistic model, strain is not explicitly defined and thus Eq. (39) cannot be directly employed for MMM. Instead, the pair distance vector is employed in the error measure, because the interatomic (pair) potential energy is a function of pair distance, which is analogous to the strain defined in continuum mechanics. We note that even for many-body potentials, the difference in each pair distance vector is also a good error indicator in potential energy. As such, the difference in strain vector field and in pair distance vector field are good error measures in energy field for the respective continuum and atomistic modeling.

We note that in many of the previous works, errors are always measured in such a way that the displacement vectors $\mathbf{U}$ or pair distance vector $\mathbf{r}_{i j}$ from different models are first computed to generate a scalar, and then the difference between the scalars is employed as the error. For instance, let $E_{\text {tot }}^{F A}$ and $\tilde{E}_{\text {tot }}$ be the respective total potential energy scalar from atomistic modeling 
and any other model generated by operations on the respective $\mathbf{r}_{i j}$ or $\mathbf{U}$ from each model, then the error is calculated as:

$$
e r r=\left|\frac{E_{t o t}^{F A}-\tilde{E}_{t o t}}{E_{t o t}^{F A}}\right|
$$

We note that this is not a good error indicator because there are cases that the difference, for instance, in displacement field is significant but err is quite small, as schematically shown in Fig. 6. In contrast, the error norms defined in Eqs. (40)-(41) can eliminate this possibility.

Then using the error norms defined in Eq. (40), the discretization error $e_{U}^{\text {disc }}$ and sampling error $e_{U}^{s a m}$ defined in Eq. (37) in displacement field can be deduced as:

$$
\begin{gathered}
e_{U}^{\text {disc }}=\left[\frac{\sum_{i \in \mathcal{N}_{A}}\left(\mathbf{u}_{i}^{\mathrm{FA}}-\mathbf{u}_{0 \mathrm{p} * \mathrm{~s}_{i}}^{\mathrm{MMM}}\right)^{\mathrm{T}}\left(\mathbf{u}_{i}^{\mathrm{FA}}-\mathbf{u}_{0 \mathrm{p} * \mathrm{~s}_{i}}^{\mathrm{MMM}}\right)}{\sum_{i \in \mathcal{N}_{A}}\left(\mathbf{u}_{i}^{\mathrm{FA}}\right)^{\mathrm{T}} \mathbf{u}_{i}^{\mathrm{FA}}}\right]^{1 / 2}=\frac{\left\|\mathbf{u}^{\mathrm{FA}}-\mathbf{U}_{0 \mathrm{p} * \mathrm{~s}}^{\mathrm{MMM}}\right\|_{2}}{\left\|\mathbf{U}^{\mathrm{FA}}\right\|_{2}} \\
e_{U}^{s a m}=\left[\frac{\sum_{i \in \mathcal{N}_{A}}\left(\mathbf{u}_{0 \mathrm{p} * \mathrm{~s}_{i}}^{\mathrm{MMM}}-\mathbf{u}_{i}^{\mathrm{MMM}}\right)^{\mathrm{T}}\left(\left(\mathbf{u}_{0 \mathrm{p} * \mathrm{~s}_{i}}^{\mathrm{MMM}}-\mathbf{u}_{i}^{\mathrm{MMM}}\right)\right)}{\sum_{i \in \mathcal{N}_{A}}\left(\mathbf{u}_{i}^{\mathrm{FA}}\right)^{\mathrm{T}} \mathbf{u}_{i}^{\mathrm{FA}}}\right]^{1 / 2}=\frac{\left\|\mathbf{U}_{0 \mathrm{p} * \mathrm{~s}}^{\mathrm{MM}}-\mathbf{U}^{\mathrm{MMM}}\right\|_{2}}{\left\|\mathbf{U}^{\mathrm{FA}}\right\|_{2}}
\end{gathered}
$$

Note that $e_{U}^{\text {total }}, e_{U}^{\text {disc }}$ and $e_{U}^{s a m}$ are all normalized by $\left\|\mathbf{U}^{\mathrm{FA}}\right\|_{2}$ such that they are comparable to each other.

Similarly, the discretization error $e_{E}^{\text {disc }}$ and sampling error $e_{E}^{s a m}$ in energy field are defined as:

$$
\begin{gathered}
\mathrm{e}_{\mathrm{E}}^{d i s c}=\left[\frac{\sum_{i \in \mathcal{N}_{\mathrm{A}}} \sum_{j \in \mathcal{N}_{i}}\left(\left(\mathrm{r}_{i j}^{\mathrm{FA}}-\mathrm{r}_{\mathrm{ij}}^{0}\right)-\left(\mathbf{r}_{0 \mathrm{p} * \mathrm{~s}_{i j}}^{\mathrm{MMM}}-\mathrm{r}_{i j}^{0}\right)\right)^{\mathrm{T}}\left(\left(\mathrm{r}_{i j}^{\mathrm{FA}}-\mathrm{r}_{i j}^{0}\right)-\left(\mathrm{r}_{0 \mathrm{p} * \mathrm{~s}_{i j}}^{\mathrm{MMM}}-\mathrm{r}_{i j}^{0}\right)\right)}{\sum_{\mathrm{i} \in \mathcal{N}_{\mathrm{A}}} \sum_{\mathrm{j} \in \mathcal{N}_{\mathrm{i}}}\left(\left(\mathrm{r}_{i j}^{\mathrm{FA}}-\mathrm{r}_{i j}^{0}\right)\right)^{\mathrm{T}}\left(\left(\mathrm{r}_{i j}^{\mathrm{FA}}-\mathrm{r}_{i j}^{0}\right)\right)}\right]^{1 / 2} \\
e_{E}^{S a m}=\left[\frac{\sum_{i \in \mathcal{N}_{A}} \sum_{j \in \mathcal{N}_{i}}\left(\left(\mathbf{r}_{0 \mathrm{p} * \mathrm{~s}_{i j}}^{\mathrm{MM}}-\mathbf{r}_{i j}^{0}\right)-\left(\mathrm{r}_{i j}^{\mathrm{MMM}}-\mathbf{r}_{i j}^{0}\right)\right)^{\mathrm{T}}\left(\left(\mathbf{r}_{0 \mathrm{p} * \mathrm{~s}_{i j}}^{\mathrm{MM}}-\mathbf{r}_{i j}^{0}\right)-\left(\mathbf{r}_{i j}^{\mathrm{MMM}}-\mathbf{r}_{i j}^{0}\right)\right)}{\sum_{i \in \mathcal{N}_{A}} \sum_{j \in \mathcal{N}_{i}}\left(\left(\mathbf{r}_{i j}^{\mathrm{FA}}-\mathbf{r}_{i j}^{0}\right)\right)^{\mathrm{T}}\left(\left(\mathbf{r}_{i j}^{\mathrm{FA}}-\mathbf{r}_{i j}^{0}\right)\right)}\right]^{1 / 2}
\end{gathered}
$$

The errors defined in this section will be employed to quantify the performance of MMM to solve surface relaxation and bending problems in 2D and 3D in Section 4. 


\section{Numerical Examples}

In this section, the aforementioned $\mathrm{SR}^{\mathrm{MMM}}\left(\mathrm{SR}^{\mathrm{B}}+\mathrm{SR}^{\mathrm{S}}\right)$ in Section 2 will be employed in $2 \mathrm{D}$ and $3 \mathrm{D}$ to solve surface relaxation and beam bending problems. The accuracy of SR ${ }^{\mathrm{MMM}}$ will be quantified by the error norms defined in Section 3. It is worth to note that the proposed $\mathrm{SR}^{\mathrm{MMM}}$ can naturally capture both in-plane and out-of-plane surface stress components. This is due to the fact that the interatomic potential employed is directly utilized to calculate the interatomic force interactions among the surface sampling atoms, as in full atomistic modeling. As such, forces from neighbors located in out-of-plane and in-plane directions are automatically considered.

\subsection{D Surface Relaxation}

In the following numerical example, the 2D triangle lattice (Fig. 7) consists of 97 by 97 atoms (9361 atoms in total) interacting via the standard Lennard-Jones (LJ) potential, i.e., $U_{L J}=4 \varepsilon\left[(\sigma / r)^{12}-(\sigma / r)^{6}\right]$ where $r$ is the interatomic distance with the initial nearest interatomic spacing $r_{0}=2^{1 / 6}$ and potential parameters $\sigma=\varepsilon=1$. The interatomic interaction is modelled up to the second nearest neighbor interaction. For boundary conditions, the lower-left corner atom is fixed in both $x$ and $y$ directions and the upper-left corner atom is fixed in $x$ direction to eliminate rigid body motion. The lattice model is coarse-grained by a regular quadrilateral mesh (dashed lines) with 164 -node quadrilateral elements. The mesh has 25 nodes or nodal atoms and takes only $0.27 \%$ over the original full atomistic (FA) degrees of freedom (DOFs), corresponding to an element size $h_{x}=24 r_{0}$ in $x$ direction and $h_{y}=12 \sqrt{3} r_{0}$ in $y$ direction. The 2D lattice model is relaxed using a non-linear Newton solver.

Since the second nearest neighbor interaction is considered, there are one surface edge and one non-bulk layer in the direction perpendicular to the bottom and top of the lattice, respectively. And there are one surface edge and three non-bulk layers in the direction perpendicular to the left and right of the lattice. As such, the surface summation rule $\mathrm{SR}^{\mathrm{S}}$ is employed for each edge and non-bulk layer and the bulk summation rule $\mathrm{SR}^{\mathrm{B}}$ is taken for the bulk region, as schematically shown in Fig. 7.

A comparison of the displacement field distributions from FA model and from the proposed MMM calculation is shown in Fig. 8. The figure clearly illustrates that the proposed MMM with $\mathrm{SR}^{\mathrm{MMM}}$ captures the displacement tendencies in $x$ (Fig. 8a and Fig. 8b) and $y$ (Fig. 8c and Fig. 8d) directions exhibited from atomistic simulation, both qualitatively and quantitatively. In order to show the capability of MMM to capture corner and edge effects, the displacement fields of the upper-right corner and the middle of the edge on the right hand side, from the respective FA and MMM models, are tabulated in Table 1, where the accuracy of MMM is demonstrated.

In addition, the different error types defined in Section 3 are presented in Table 2. As mentioned in Section 3, the discretization error is fixed for a given mesh. Thus the sampling error is a good (if not best) indicator about the performance of a proposed summation rule. In general, to minimize the total error $e_{U}^{\text {total }}$, the sampling error $e_{U}^{\text {sam }}$ has to be minimized. As can be seen from Table 2, the introduced $e_{U}^{s a m}$ and $e_{E}^{s a m}$ are $0.04 \%$ and $0.0006 \%$ in displacement and energy field, respectively, with the proposed $\mathrm{SR}^{\mathrm{MMM}}$. The small sampling errors obtained prove that MMM has great performance. 


\subsection{D Beam Bending}

The 2D atomic beam (Fig. 9) bending problem employed in $[57,58]$ is taken here to show the effectiveness of the proposed summation rule with respect to 8-node quadratic quadrilateral element that is suitable for bending analysis. The beam model consists of 97 by 25 atoms $(2,413$ atoms in total), placed on a triangular lattice, and undergoes a deformation defined by Eqs. (47) and (48) in $x$ and $y$ directions, respectively:

$$
\begin{gathered}
u(x, y)=-\frac{P}{6 E I}\left[(6 L-3 x) x+(2+v)\left(y^{2}-\frac{D^{2}}{4}\right)\right] \\
v(x, y)=-\frac{P}{6 E I}\left[3 v y^{2}(L-x)+(4+5 v) \frac{D^{2} x}{4}+(3 L-x) x^{2}\right]
\end{gathered}
$$

where $L$ and $D$ are the length and height of the beam, respectively. Parameters in Eqs. (47) and (48) are taken as $P=1000, E=3 \times 10^{5}, v=0.3$ and $I=\frac{D^{3}}{12}$. The Lennard-Jones potential defined in Section 4.1 is taken here to describe the interatomic interaction. For boundary conditions, the atomic beam is fixed at the left lower and upper corners in $x$ direction and at the middle of the left edge in $y$ direction.

The 8-node quadratic quadrilateral element is employed to coarse grain the beam with an element size $h_{x}=12 r_{0}$ in $x$ direction and $h_{y}=6 \sqrt{3} r_{0}$ in $y$ direction. $h_{x}$ and $h_{y}$ denote the nearest nodal spacing in each direction. The coarse-grained model has 23 nodes, corresponding to $0.95 \%$ over the original FA DOFs. In order to show the significance to capture surface effect, MMM with $\mathrm{SR}^{\mathrm{B}}$ only and with $\left(\mathrm{SR}^{\mathrm{B}}+\mathrm{SR}^{\mathrm{S}}\right)$ are respectively applied to the coarse-grained model, as shown in Figs. 9a and 9b.

Figures 10a, 10b and 10c show the respective vertical bending distribution from $\mathrm{FA}, \mathrm{SR}^{\mathrm{B}}$ only and $\left(\mathrm{SR}^{\mathrm{B}}+\mathrm{SR}^{\mathrm{S}}\right)$. As seen in Fig. $10,\left(\mathrm{SR}^{\mathrm{B}}+\mathrm{SR}^{\mathrm{S}}\right)$ has much better agreement with FA results than $\mathrm{SR}^{\mathrm{B}}$ only. Table 3 quantifies the defined different errors types from different sampling approaches. As observed in Table 3, the introduced sampling error $e_{U}^{s a m}$ from $\mathrm{SR}^{\mathrm{B}}$ only and $\left(\mathrm{SR}^{\mathrm{B}}+\mathrm{SR}^{\mathrm{S}}\right)$ are $28.25 \%$ and $1.06 \%$, respectively. This observation justifies the significance of capturing surface effect and that $\mathrm{SR}^{\mathrm{S}}$ can effectively capture surface effect with respect to the employed quadratic element for bending analysis.

\subsection{D Surface Relaxation}

The numerical example considered next is the free surface relaxation of an FCC crystal lattice. The FCC lattice consists of 24 by 24 by 24 unit cells in the $x, y$ and $z$ directions, corresponding to 58,825 atoms as shown in Fig. 11. The same interatomic potential employed in 2D surface relaxation example is also used here. The atomic interaction is truncated after the third shell of the nearest neighbors. To eliminate rigid body motions, the boundary conditions in [39] are employed. Let the cubic center be the origin and $L$ be the length of the cube edge. The corner at $(-L / 2,-L / 2,-L / 2)$ is fixed in all directions, the corner at $(L / 2,-L / 2,-L / 2)$ is fixed in $y$ and $z$ directions, and the corner at $(-L / 2, L / 2,-L / 2)$ is fixed in the $z$ direction. 
To coarse grain the FCC lattice, a regular hexahedral mesh (dashed lines) with 27 8-node hexahedral elements is introduced, as shown in Fig. 11. The mesh has 64 nodal atoms with an element size $h=8 \sqrt{2} r_{0}$ in each direction, which takes only $0.11 \%$ of the original full atomistic (FA) DOFs. Since the atomic interaction is modelled up to the third shell of nearest neighbors, there is one free surface and one non-bulk layer in the direction perpendicular to each cubic side surface, as shown in Fig.12. Then $\mathrm{SR}^{\mathrm{S}}$ is applied to both the free surface and the non-bulk layer, as illustrated in Fig. 11b. Note that $\mathrm{SR}^{\mathrm{S}}$ applied to the non-bulk layer is invisible in Fig. 11. The $\mathrm{SR}^{\mathrm{B}}$ employed is schematically shown in Fig. 13, as mentioned in Section 2.

Figures 14-16 show the displacement distribution comparison between FA modeling and MMM simulation. As can be seen, MMM accurately captures the distribution trend in each (positive or negative) direction, similar to what has been observed in the $2 \mathrm{D}$ case. The expansion or contraction of a surface is clearly matched in $x, y$ and $z$ directions. In order to compare the distribution inside the cube, the models in Figs. 14-16 are cut through by $x=0, y=0$ and $z=0$ planes, respectively. As shown in Figs. 17-19, very good agreement has been achieved. The purpose of showing the distribution pattern in each direction is to demonstrate that MMM has uniform accuracy in each direction and no direction preference is observed, as will be shown in Table 4.

The displacement fields for the corner at $(L / 2, L / 2, L / 2)$, the atom at the middle of the edge connecting corners at $(L / 2,-L / 2, L / 2)$ and $(L / 2, L / 2, L / 2)$, and the atom at the center of $+x$ free surface are presented in Table 4 to show the capability of MMM to capture the corner, edge and surface effects in 3D. The uniform accuracy of MMM in each direction is clearly demonstrated in Table 4.

To more rigorously understand the performance of MMM in 3D and to show the importance of accurately capturing surface effect, $e_{U}^{\text {total }}, e_{U}^{\text {disc }}, e_{U}^{\text {sam }}$ and $e_{E}^{s a m}$ from MMM with $\mathrm{SR}^{\mathrm{B}}$ only (Fig. 11a) and with $\left(\mathrm{SR}^{\mathrm{B}}+\mathrm{SR}^{\mathrm{S}}\right)$ (Fig. $\left.11 \mathrm{~b}\right)$ are presented in Table 5. The introduced $e_{U}^{s a m}$ with $\mathrm{SR}^{\mathrm{B}}$ only and with $\left(\mathrm{SR}^{\mathrm{B}}+\mathrm{SR}^{\mathrm{S}}\right)$ are $44.53 \%$ and $0.71 \%$, respectively, which shows the effectiveness of the proposed $\mathrm{SR}^{\mathrm{S}}$ to capture surface effect. As what has been observed in $2 \mathrm{D}$, the good performance of $\mathrm{MMM}$ lies in that $\mathrm{SR}^{\mathrm{MMM}}$ accurately represents and reproduces the energy distribution of coarse-grained atomistic model with solid mathematical foundation.

It is worth to note that the employed 2D triangular and 3D FCC lattices are both centrosymmetric. The application of $\mathrm{SR}^{\mathrm{MMM}}$ to non-centrosymmetric lattices such as Silicon will be reported in our future publications. For example, techniques proposed in [41, 73-75] may be adopted into the proposed $\mathrm{SR}^{\mathrm{MMM}}$ to consider the shift vectors or internal degrees of freedom coming with noncentrosymmetric lattices.

Compared to full molecular mechanics, the efficiency of MMM is indirectly measured by the number of DOFs employed in this work. The additional computational effort required in MMM, such as the calculation of weights for primary sampling atoms, makes the computational cost reduction not proportional to the reduction in DOFs. However, the efficiency of MMM is not affected significantly since the extra computational cost in MMM takes a small portion of the overall computational effort, when compared to the computational time required to, for instance, 
solve nonlinear governing equations. In fact, the efficiency of MMM based on linear elements has been reported in [67] for problems such as nano-indentation. A more comprehensive study on the efficiency of MMM will be investigated in the future since efficiency is as important as accuracy. 


\section{Conclusions}

We have proposed the surface summation rule $\mathrm{SR}^{\mathrm{S}}$ within the framework of multiresolution molecular mechanics (MMM). Together with the previously developed bulk summation rule $\mathrm{SR}^{\mathrm{B}}$, $\mathrm{SR}^{\mathrm{MMM}}$ is completed. Analogous to classical quadrature rules to evaluate energy integral in conventional continuum mechanics, $\mathrm{SR}^{\mathrm{MMM}}$ is based on mathematically deriving the order or distribution of finite energy summations involved in coarse-grained atomistic model and then determines the number, positions and weights for quadrature-type (sampling) atoms. $\mathrm{SR}^{\mathrm{S}}$ and $\mathrm{SR}^{\mathrm{B}}$ are designed correspondingly to treat surface and bulk regions respectively based on the observation that the surface energy distribution is different from bulk energy map mathematically. Physically, the difference is due to the fact that surface atoms lack bonding neighbors and are not fully coordinated compared with their bulk counterpart.

To analyze the performance of any summation rule, different error sources are identified and the total error is decomposed into discretization error and sampling error. It is pointed out that sampling error is a good indicator of the accuracy of any proposed summation rule since discretization error is fixed for a given mesh. To quantify different error sources, rigorous errors norms, as inspired by the norms to quantify FEM accuracy in continuum mechanics, are defined.

Numerical examples in 2D and 3D have been employed to verify and validate MMM with $\mathrm{SR}^{\mathrm{MMM}}$. For both the 2D triangle lattices and 3D FCC model, the proposed MMM qualitatively and quantitatively captures the mechanical response as demonstrated in the two surface relaxations and one beam bending examples. The contraction or expansion of a surface is clearly matched compared to the FA simulation. A comprehensive comparison between displacement results from FA simulation and MMM shows similar and uniform accuracy of MMM in $x, y$ and $z$ directions. Corner and edge effects are also accurately captured. The effectiveness of SR ${ }^{\text {MMM }}$ with respect to high order element is demonstrated by employing the quadratic quadrilateral element to solve a beam bending problem. Furthermore, the proposed $\mathrm{SR}^{\mathrm{MMM}}$ introduces very small sampling errors as quantified by the error norms defined in both the displacement and energy fields.

In the future, several research directions will be pursued. First, the embedded atom (EAM) potential will be employed so as to apply MMM to realistic metallic materials. Note that the framework of MMM is potential-independent (as long as the potential can be well-approximated by a harmonic potential within the convex region). Second, we will apply MMM to models with complex geometries such as rough surfaces to study the effect of surface orientations. Finally, surface-defect interaction will be considered by using atomistic simulation to locally track defect at where it is needed. 


\section{Acknowledgement}

Financial support from the National Science Foundation (CMMI-1334763) is gratefully acknowledged. 


\section{Figure Captions}

Figure 1 Schematically overview of the MMM methodology in analogy to Finite Element Method (FEM): FEM for continuum modeling (a) and MMM for discrete modeling (b). The meaning of different symbols are explained in Section 2.1

Figure 2 Schematically illustration of energy distributions for coarse-grained continuum model (a) and coarse-grained atomistic model (b). Solid line denotes a continuous curve and dashed line a curve passing through discrete energy point for each atom. The solid $x$ axis means $x_{c}$ continuously spans the continuum domain (a) and the dashed $x$ axis represents that $x_{a}$ discretely spans the space (b).

Figure 3 Energy distribution for atoms in bulk region and atoms in surface areas

Figure 4 Employed $\mathrm{SR}^{\mathrm{MMM}}\left(\mathrm{SR}^{\mathrm{B}}+\mathrm{SR}^{\mathrm{S}}\right)$ in $2 \mathrm{D}$ for the selected 4-node quadrilateral element: $\mathrm{SR}^{\mathrm{MMM}}$ for an element that contains a corner atom (a); $\mathrm{SR}^{\mathrm{MMM}}$ for an element that contains only one surface edge (b); $\mathrm{SR}^{\mathrm{MMM}}$ for an element that contains a corner atom and non-bulk layers (dashed line) (c); $\mathrm{SR}^{\mathrm{MMM}}$ for an element that contains one surface edge and one non-bulk layer (d). Dashed rectangle represents the bulk region. $\mathrm{SR}^{\mathrm{B}}$ is employed for bulk non-sampling atoms (NSAs) and $\mathrm{SR}^{\mathrm{S}}$ for surface and non-bulk NSAs

Figure 5 Schematically demonstration of error sources and types: Model A: full atomistic model; Model B: a general MMM model with $\mathrm{SR}^{\mathrm{MMM}}$; Model C: a specifically designed model where the energy of each atom is explicitly considered. The distance between Modes A and B is the total error; distance between Models $\mathrm{A}$ and $\mathrm{C}$ is the discretization error; distance between Models $\mathrm{C}$ and $\mathrm{B}$ is the sampling error. Discretization error is fixed for a given mesh.

Figure 6 Schematically illustration of the possibility in an error estimator that the distance between different displacement fields could be very large even though they produce the same potential energy scalar $E_{0}: \mathbf{U}^{\mathrm{FA}}$ is from full atomistic simulation and $\widetilde{\mathbf{U}}$ from any other approximation.

Figure 7 The employed 4-node quadrilateral mesh (dashed line) and summation rule $\mathrm{SR}^{\mathrm{MMM}}$ for the $2 \mathrm{D}$ surface relaxation problem: $\mathrm{SR}^{\mathrm{B}}$ for the bulk region and $\mathrm{SR}^{\mathrm{S}}$ for the surface and non-bulk regions

Figure 8 Comparison of displacement distributions from full atomistic (FA) calculation and MMM: the $x$-displacement distributions from FA (a) and MMM (b); the $y$-displacement distributions from FA (c) and MMM (d)

Figure 9 The employed 8-node quadratic quadrilateral mesh (dashed line) with $\mathrm{SR}^{\mathrm{B}}$ only (a) and with $\left(\mathrm{SR}^{\mathrm{S}}+\mathrm{SR}^{\mathrm{B}}\right)(\mathrm{b})$ for the $2 \mathrm{D}$ beam bending problem

Figure 10 Comparison of vertical bending distributions from full atomistic (FA) (a), $\mathrm{SR}^{\mathrm{B}}$ only (b) and $\left(\mathrm{SR}^{\mathrm{S}}+\mathrm{SR}^{\mathrm{B}}\right)(\mathrm{c})$

Figure 11 The employed 8-node hexahedral mesh (dashed line) for the $3 \mathrm{D}$ problem: $\mathrm{SR}^{\mathrm{B}}$ only is employed in (a) and $\left(\mathrm{SR}^{\mathrm{S}}+\mathrm{SR}^{\mathrm{B}}\right)$ is used in (b). The blue dots on the free surfaces and edges (b) 
shows the applied $\mathrm{SR}^{\mathrm{S}} ; \mathrm{SR}^{\mathrm{S}}$ and $\mathrm{SR}^{\mathrm{B}}$ are also utilized for the non-bulk and bulk layers, respectively, and are invisible

Figure 12 The respective free surface, non-bulk and bulk layers of the $3 \mathrm{D}$ example: $\mathrm{SR}^{\mathrm{S}}$ is applied to both free surface and non-bulk layers and $\mathrm{SR}^{\mathrm{B}}$ is employed for bulk layers

Figure 13 Schematically demonstration of the employed $\mathrm{SR}^{\mathrm{B}}$ with 8-node hexahedral element for bulk layers: red dots are the nodal atoms and blue dots represent the selected primary sampling atoms (PSAs)

Figure 14 Comparison of $x$-displacement distributions on surfaces from full atomistics (FA) and MMM: $+x$-displacement from FA (a) and MMM (b); $-x$-displacement from FA (c) and MMM (d)

Figure 15 Comparison of $y$-displacement distributions on surfaces from full atomistics (FA) and MMM: $+y$-displacement from FA (a) and MMM (b); - $y$-displacement from FA (c) and MMM (d)

Figure 16 Comparison of $z$-displacement distributions on surfaces from full atomistics (FA) and MMM: +z-displacement from FA (a) and MMM (b); -z-displacement from FA (c) and MMM (d)

Figure 17 Comparison of $x$-displacement distributions from bulk along the plane $x=0$ from full atomistics (FA) (a) and MMM (b)

Figure 18 Comparison of $y$-displacement distributions from bulk along the plane $y=0$ from full atomistics (FA) (a) and MMM (b)

Figure 19 Comparison of $z$-displacement distributions from bulk along the plane $z=0$ from full atomistics (FA) (a) and MMM (b) 


\section{References}

[1] E.W. Wong, P.E. Sheehan, C.M. Lieber, Nanobeam mechanics: elasticity, strength, and toughness of nanorods and nanotubes, Science, 277 (1997) 1971-1975.

[2] P. Poncharal, Z. Wang, D. Ugarte, W.A. De Heer, Electrostatic deflections and electromechanical resonances of carbon nanotubes, Science, 283 (1999) 1513-1516.

[3] S. Cuenot, C. Frétigny, S. Demoustier-Champagne, B. Nysten, Surface tension effect on the mechanical properties of nanomaterials measured by atomic force microscopy, Physical Review B, 69 (2004) 165410.

[4] R. Agrawal, B. Peng, E.E. Gdoutos, H.D. Espinosa, Elasticity size effects in ZnO nanowiresa combined experimental-computational approach, Nano Letters, 8 (2008) 3668-3674.

[5] A. Asthana, K. Momeni, A. Prasad, Y. Yap, R. Yassar, In situ observation of size-scale effects on the mechanical properties of ZnO nanowires, Nanotechnology, 22 (2011) 265712.

[6] J.-Y. Kim, D. Jang, J.R. Greer, Crystallographic orientation and size dependence of tensioncompression asymmetry in molybdenum nano-pillars, International Journal of Plasticity, 28 (2012) 46-52.

[7] Y. Zhu, Q. Qin, F. Xu, F. Fan, Y. Ding, T. Zhang, B.J. Wiley, Z.L. Wang, Size effects on elasticity, yielding, and fracture of silver nanowires: In situ experiments, Physical review B, 85 (2012) 045443.

[8] G. Jing, H.L. Duan, X. Sun, Z. Zhang, J. Xu, Y. Li, J. Wang, D. Yu, Surface effects on elastic properties of silver nanowires: contact atomic-force microscopy, Physical Review B, 73 (2006) 235409.

[9] R.E. Miller, V.B. Shenoy, Size-dependent elastic properties of nanosized structural elements, Nanotechnology, 11 (2000) 139.

[10] G. Cao, X. Chen, Size dependence and orientation dependence of elastic properties of $\mathrm{ZnO}$ nanofilms, International journal of solids and structures, 45 (2008) 1730-1753.

[11] X. Zhou, H. Zhou, X. Li, C. Chen, Size effects on tensile and compressive strengths in metallic glass nanowires, Journal of the Mechanics and Physics of Solids, 84 (2015) 130-144.

[12] H. Liang, M. Upmanyu, H. Huang, Size-dependent elasticity of nanowires: nonlinear effects, Physical Review B, 71 (2005) 241403.

[13] V.B. Shenoy, Atomistic calculations of elastic properties of metallic fcc crystal surfaces, Physical Review B, 71 (2005) 094104.

[14] L. Zhou, H. Huang, Are surfaces elastically softer or stiffer?, Applied Physics Letters, 84 (2004) 1940-1942.

[15] A. To, J. Tao, M. Kirca, L. Schalk, Ligament and joint sizes govern softening in nanoporous aluminum, Applied physics letters, 98 (2011) 051903.

[16] B. Arash, J.-W. Jiang, T. Rabczuk, A review on nanomechanical resonators and their applications in sensors and molecular transportation, Applied Physics Reviews, 2 (2015) 021301. [17] K. Eom, H.S. Park, D.S. Yoon, T. Kwon, Nanomechanical resonators and their applications in biological/chemical detection: nanomechanics principles, Physics Reports, 503 (2011) 115163.

[18] J. Chaste, A. Eichler, J. Moser, G. Ceballos, R. Rurali, A. Bachtold, A nanomechanical mass sensor with yoctogram resolution, Nature nanotechnology, 7 (2012) 301-304.

[19] M. Hanay, S. Kelber, A. Naik, D. Chi, S. Hentz, E. Bullard, E. Colinet, L. Duraffourg, M. Roukes, Single-protein nanomechanical mass spectrometry in real time, Nature nanotechnology, 7 (2012) 602-608. 
[20] J. Moser, J. Güttinger, A. Eichler, M.J. Esplandiu, D. Liu, M. Dykman, A. Bachtold, Ultrasensitive force detection with a nanotube mechanical resonator, Nature nanotechnology, 8 (2013) 493-496.

[21] X. Feng, C. White, A. Hajimiri, M.L. Roukes, A self-sustaining ultrahigh-frequency nanoelectromechanical oscillator, Nature nanotechnology, 3 (2008) 342-346.

[22] L.G. Villanueva, R.B. Karabalin, M.H. Matheny, E. Kenig, M.C. Cross, M.L. Roukes, A nanoscale parametric feedback oscillator, Nano letters, 11 (2011) 5054-5059.

[23] X. Feng, M. Matheny, C. Zorman, M. Mehregany, M. Roukes, Low voltage

nanoelectromechanical switches based on silicon carbide nanowires, Nano letters, 10 (2010)

2891-2896.

[24] R. Dingreville, J. Qu, M. Cherkaoui, Surface free energy and its effect on the elastic behavior of nano-sized particles, wires and films, Journal of the Mechanics and Physics of Solids, 53 (2005) 1827-1854.

[25] J.-G. Guo, Y.-P. Zhao, The size-dependent bending elastic properties of nanobeams with surface effects, Nanotechnology, 18 (2007) 295701.

[26] C. Lim, L. He, Size-dependent nonlinear response of thin elastic films with nano-scale thickness, International Journal of Mechanical Sciences, 46 (2004) 1715-1726.

[27] L. He, C. Lim, B. Wu, A continuum model for size-dependent deformation of elastic films of nano-scale thickness, International Journal of Solids and Structures, 41 (2004) 847-857.

[28] H. Zhu, B.L. Karihaloo, Size-dependent bending of thin metallic films, International Journal of Plasticity, 24 (2008) 991-1007.

[29] C. Chen, Y. Shi, Y. Zhang, J. Zhu, Y. Yan, Size dependence of Young's modulus in ZnO nanowires, Physical review letters, 96 (2006) 075505.

[30] J. He, C.M. Lilley, Surface effect on the elastic behavior of static bending nanowires, Nano Letters, 8 (2008) 1798-1802.

[31] R.C. Cammarata, Surface and interface stress effects in thin films, Progress in surface science, 46 (1994) 1-38.

[32] H. Duan, J. Wang, Z. Huang, B. Karihaloo, Size-dependent effective elastic constants of solids containing nano-inhomogeneities with interface stress, Journal of the Mechanics and Physics of Solids, 53 (2005) 1574-1596.

[33] A. Javili, A. McBride, P. Steinmann, Thermomechanics of solids with lower-dimensional energetics: on the importance of surface, interface, and curve structures at the nanoscale. A unifying review, Applied Mechanics Reviews, 65 (2013) 010802.

[34] A. Javili, P. Steinmann, A finite element framework for continua with boundary energies. Part III: The thermomechanical case, Computer Methods in Applied Mechanics and Engineering, 200 (2011) 1963-1977.

[35] A. Javili, P. Steinmann, A finite element framework for continua with boundary energies.

Part II: The three-dimensional case, Computer Methods in Applied Mechanics and Engineering, 199 (2010) 755-765.

[36] A. Javili, P. Steinmann, A finite element framework for continua with boundary energies. Part I: The two-dimensional case, Computer Methods in Applied Mechanics and Engineering, 198 (2009) 2198-2208.

[37] M. Farsad, F.J. Vernerey, H.S. Park, An extended finite element/level set method to study surface effects on the mechanical behavior and properties of nanomaterials, International Journal for Numerical Methods in Engineering, 84 (2010) 1466-1489. 
[38] J. Yvonnet, H.L. Quang, Q.-C. He, An XFEM/level set approach to modelling surface/interface effects and to computing the size-dependent effective properties of nanocomposites, Computational Mechanics, 42 (2008) 119-131.

[39] H.S. Park, P.A. Klein, G.J. Wagner, A surface Cauchy-Born model for nanoscale materials, International Journal for Numerical Methods in Engineering, 68 (2006) 1072-1095.

[40] H.S. Park, P.A. Klein, Surface Cauchy-Born analysis of surface stress effects on metallic nanowires, Physical Review B, 75 (2007) 085408.

[41] H.S. Park, P.A. Klein, A surface Cauchy-Born model for silicon nanostructures, Computer methods in applied mechanics and engineering, 197 (2008) 3249-3260.

[42] M. Qomi, A. Aghaei, A. Khoei, Multi - scale modeling of surface effect via the boundary Cauchy - Born method, International journal for numerical methods in engineering, 85 (2011) 827-846.

[43] A. Khoei, A. Aramoon, A multi-scale modeling of surface effect via the modified boundary Cauchy-Born model, Materials Science and Engineering: C, 32 (2012) 1993-2000.

[44] Q. Yang, E. Biyikli, P. Zhang, R. Tian, A.C. To, Atom collocation method, Computer Methods in Applied Mechanics and Engineering, 237 (2012) 67-77.

[45] J.S. Amelang, D.M. Kochmann, Surface effects in nanoscale structures investigated by a fully-nonlocal energy-based quasicontinuum method, Mechanics of Materials, (2015).

[46] J. Knap, M. Ortiz, An analysis of the quasicontinuum method, Journal of the Mechanics and Physics of Solids, 49 (2001) 1899-1923.

[47] B. Eidel, A. Stukowski, A variational formulation of the quasicontinuum method based on energy sampling in clusters, Journal of the Mechanics and Physics of Solids, 57 (2009) 87-108. [48] Q. Yang, E. Biyikli, A.C. To, Multiresolution molecular mechanics: statics, Computer Methods in Applied Mechanics and Engineering, 258 (2013) 26-38.

[49] M.E. Gurtin, A.I. Murdoch, A continuum theory of elastic material surfaces, Archive for Rational Mechanics and Analysis, 57 (1975) 291-323.

[50] G. Yun, H.S. Park, Surface stress effects on the bending properties of fcc metal nanowires, Physical Review B, 79 (2009) 195421.

[51] H.S. Park, P.A. Klein, Surface stress effects on the resonant properties of metal nanowires: the importance of finite deformation kinematics and the impact of the residual surface stress, Journal of the Mechanics and Physics of Solids, 56 (2008) 3144-3166.

[52] M.N. Esfahani, M.R. Sonne, J.H. Hattel, B.E. Alaca, Thermo-coupled Surface CauchyBorn theory: An engineering finite element approach to modeling of nanowire thermomechanical response, Mechanics of Materials, 94 (2016) 46-52.

[53] L. Beex, R. Peerlings, M. Geers, A quasicontinuum methodology for multiscale analyses of discrete microstructural models, International Journal for Numerical Methods in Engineering, 87 (2011) 701-718.

[54] L. Beex, R. Peerlings, M. Geers, A multiscale quasicontinuum method for lattice models with bond failure and fiber sliding, Computer Methods in Applied Mechanics and Engineering, 269 (2014) 108-122.

[55] L. Beex, R. Peerlings, M. Geers, A multiscale quasicontinuum method for dissipative lattice models and discrete networks, Journal of the Mechanics and Physics of Solids, 64 (2014) 154169.

[56] J. Amelang, G. Venturini, D. Kochmann, Summation rules for a fully nonlocal energy-based quasicontinuum method, Journal of the Mechanics and Physics of Solids, (2015). 
[57] Q. Yang, A.C. To, Multiresolution molecular mechanics: A unified and consistent framework for general finite element shape functions, Computer Methods in Applied Mechanics and Engineering, 283 (2015) 384-418.

[58] Q. Yang, E. Biyikli, A.C. To, Multiresolution molecular mechanics: convergence and error structure analysis, Computer Methods in Applied Mechanics and Engineering, 269 (2014) 20-45. [59] E. Biyikli, Q. Yang, A.C. To, Multiresolution molecular mechanics: dynamics, Computer methods in applied mechanics and engineering, 274 (2014) 42-55.

[60] S. Kwon, Y. Lee, J.Y. Park, D. Sohn, J.H. Lim, S. Im, An efficient three-dimensional adaptive quasicontinuum method using variable-node elements, Journal of Computational Physics, 228 (2009) 4789-4810.

[61] L. Beex, O. Rokoš, J. Zeman, S. Bordas, Higher - order quasicontinuum methods for elastic and dissipative lattice models: uniaxial deformation and pure bending, GAMM - Mitteilungen, 38 (2015) 344-368.

[62] L. Beex, P. Kerfriden, T. Rabczuk, S. Bordas, Quasicontinuum-based multiscale approaches for plate-like beam lattices experiencing in-plane and out-of-plane deformation, Computer Methods in Applied Mechanics and Engineering, 279 (2014) 348-378.

[63] H. Duan, J. Wang, B.L. Karihaloo, Theory of elasticity at the nanoscale, Advances in applied mechanics, 42 (2009) 1-68.

[64] J. Wang, Z. Huang, H. Duan, S. Yu, X. Feng, G. Wang, W. Zhang, T. Wang, Surface stress effect in mechanics of nanostructured materials, Acta Mechanica Solida Sinica, 24 (2011) 52-82. [65] A.M. Abazari, S.M. Safavi, G. Rezazadeh, L.G. Villanueva, Modelling the Size Effects on the Mechanical Properties of Micro/Nano Structures, Sensors, 15 (2015) 28543-28562.

[66] E. Biyikli, A.C. To, Multiresolution molecular mechanics: Adaptive analysis, Computer Methods in Applied Mechanics and Engineering, 305 (2016) 682-702.

[67] E. Biyikli, A.C. To, Multiresolution molecular mechanics: Implementation and efficiency, Journal of Computational Physics, (2016).

[68] Y. Kulkarni, J. Knap, M. Ortiz, A variational approach to coarse graining of equilibrium and non-equilibrium atomistic description at finite temperature, Journal of the Mechanics and Physics of Solids, 56 (2008) 1417-1449.

[69] W.K. Kim, M. Luskin, D. Perez, A. Voter, E. Tadmor, Hyper-QC: An accelerated finitetemperature quasicontinuum method using hyperdynamics, Journal of the Mechanics and Physics of Solids, 63 (2014) 94-112.

[70] E. Tadmor, F. Legoll, W. Kim, L. Dupuy, R. Miller, Finite-temperature quasi-continuum, Applied Mechanics Reviews, 65 (2013) 010803.

[71] L.M. Dupuy, E.B. Tadmor, R.E. Miller, R. Phillips, Finite-temperature quasicontinuum: Molecular dynamics without all the atoms, Physical Review Letters, 95 (2005) 060202.

[72] O. Zienkiewicz, R. Taylor, J. Zhu, The finite element method: its basis and fundamentals. 2005, in, Butterworth-Heinemann, 2005.

[73] P. Zhang, Y. Huang, P. Geubelle, P. Klein, K. Hwang, The elastic modulus of single-wall carbon nanotubes: a continuum analysis incorporating interatomic potentials, International Journal of Solids and Structures, 39 (2002) 3893-3906.

[74] E. Tadmor, G. Smith, N. Bernstein, E. Kaxiras, Mixed finite element and atomistic formulation for complex crystals, Physical Review B, 59 (1999) 235.

[75] V. Sorkin, R. Elliott, E. Tadmor, A local quasicontinuum method for 3D multilattice crystalline materials: Application to shape-memory alloys, Modelling and Simulation in Materials Science and Engineering, 22 (2014) 055001. 

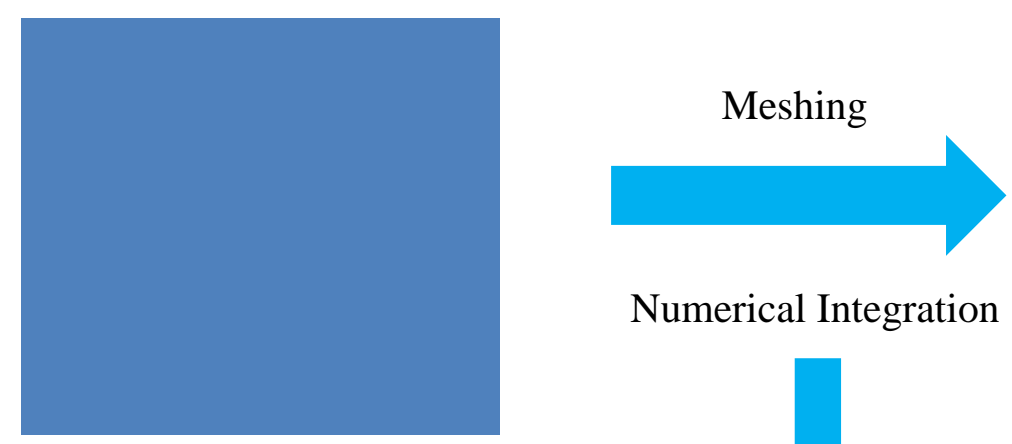

Numerical Integration

Continuum Modeling

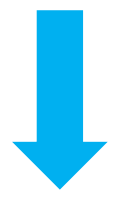

$$
E_{c}=\int_{\Omega^{c}} W d \Omega^{c} \cong \tilde{E}_{c}=\sum_{i \in \mathcal{N}_{Q}} w_{i}^{c} W_{i}^{\mathrm{Q}}
$$

Quadrature point

(a)

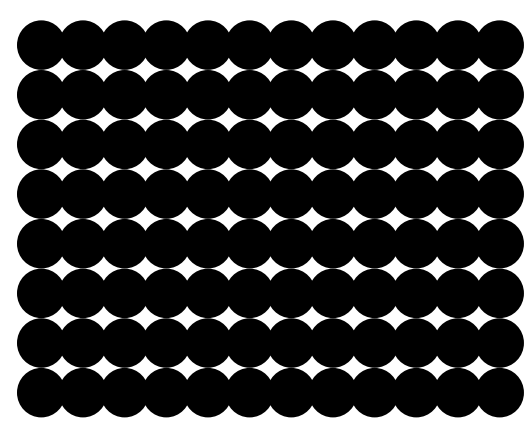

Atomistic Modeling

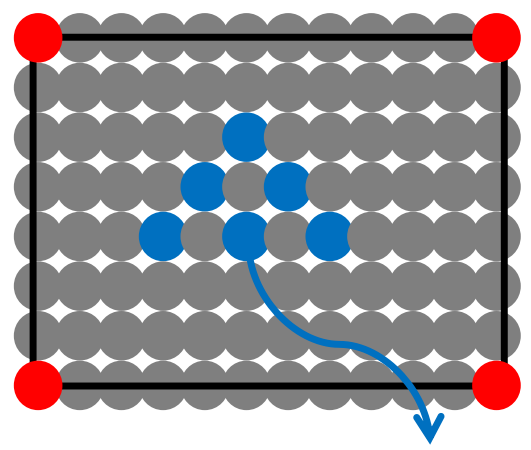

Quadrature-type or sampling atom $E_{a}=\sum_{i \in \mathcal{N}_{A}} E_{i} \cong \tilde{E}_{a}=\sum_{i \in \mathcal{N}_{S}} w_{i}^{a} E_{i}$

(b)

Figure 1 Schematically overview of the MMM methodology in analogy to Finite Element Method (FEM): FEM for continuum modeling (a) and MMM for discrete modeling (b). The meaning of different symbols are explained in Section 2.1 

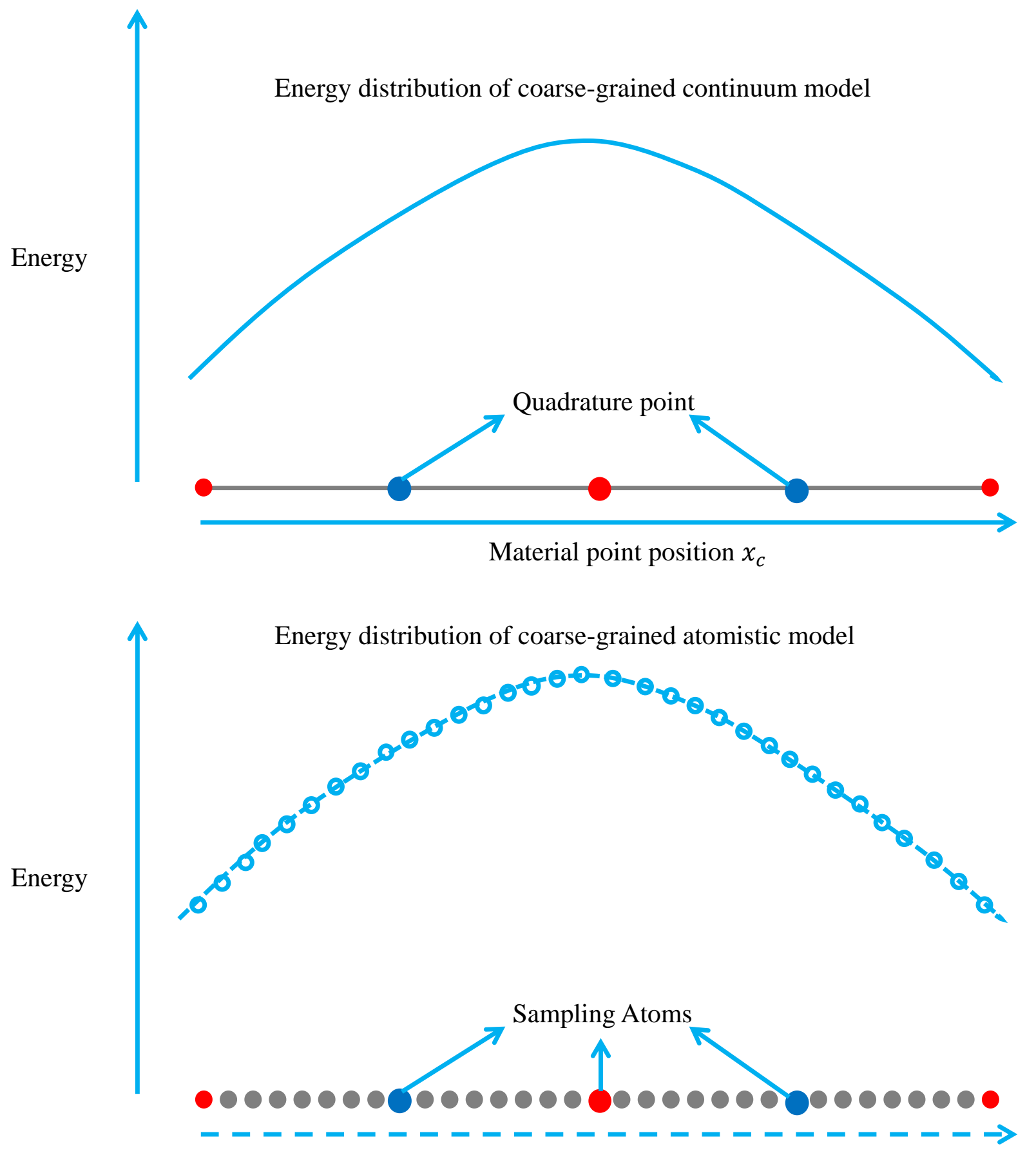

Atomic position $x_{a}$

Figure 2 Schematically illustration of energy distributions for coarse-grained continuum model (a) and coarse-grained atomistic model (b). Solid line denotes a continuous curve and dashed line a curve passing through discrete energy point for each atom. The solid $x$ axis means $x_{c}$ continuously spans the continuum domain (a) and the dashed $x$ axis represents that $x_{a}$ discretely spans the space (b). 


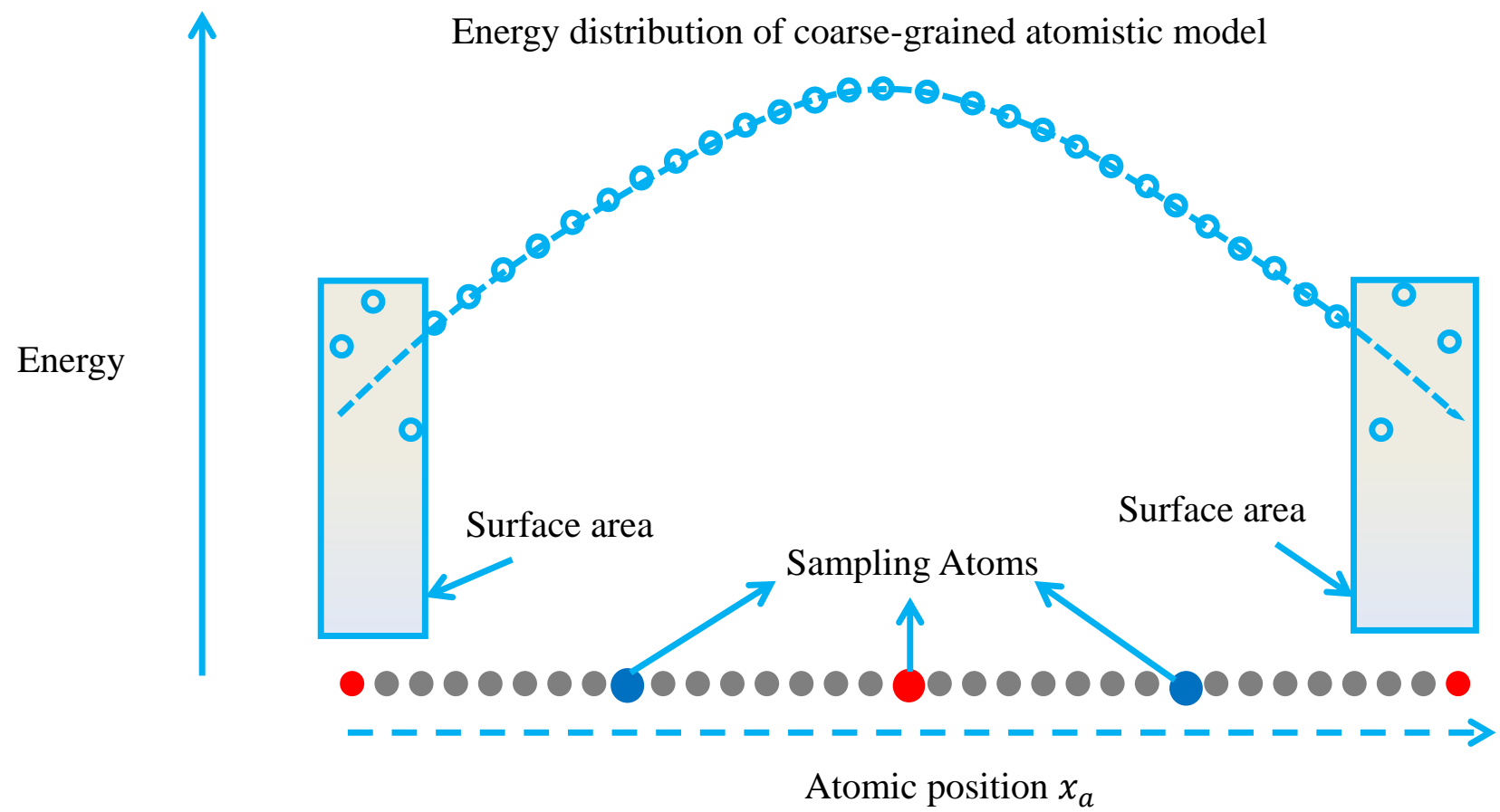

Figure 3 Energy distribution for atoms in bulk region and atoms in surface areas 


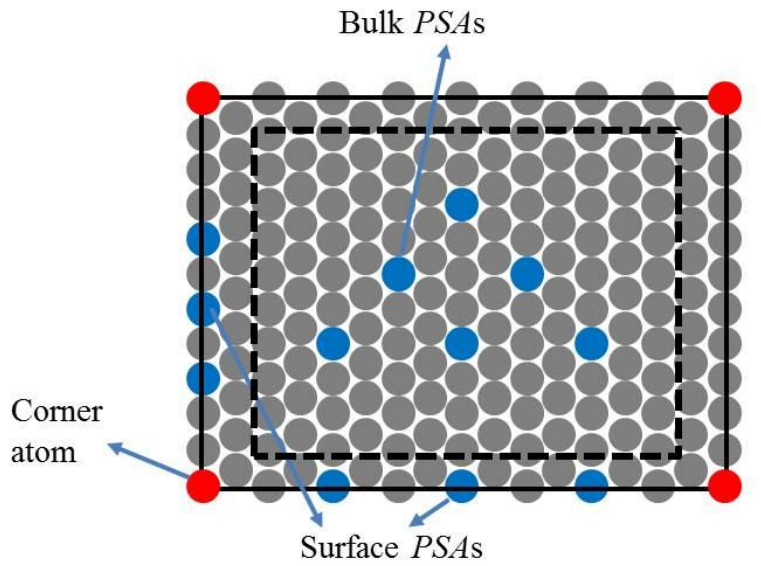

(a)

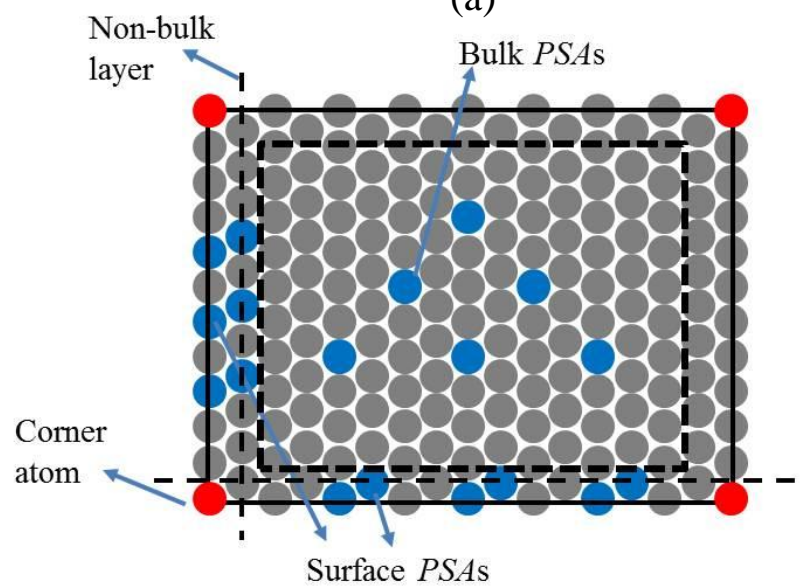

(c)

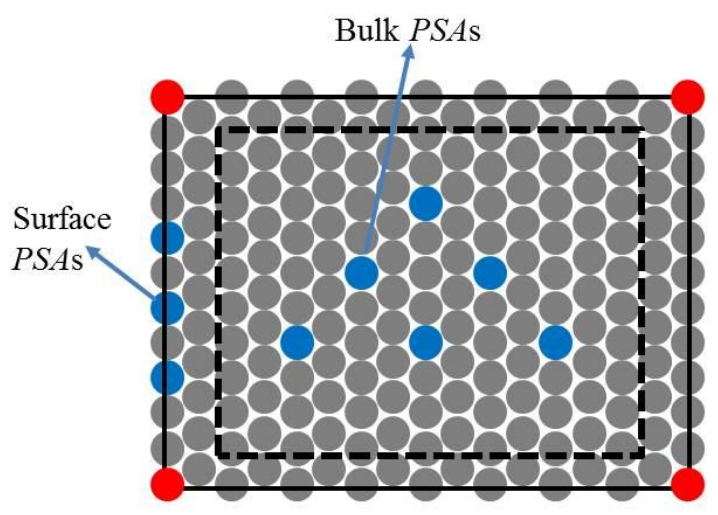

(b)

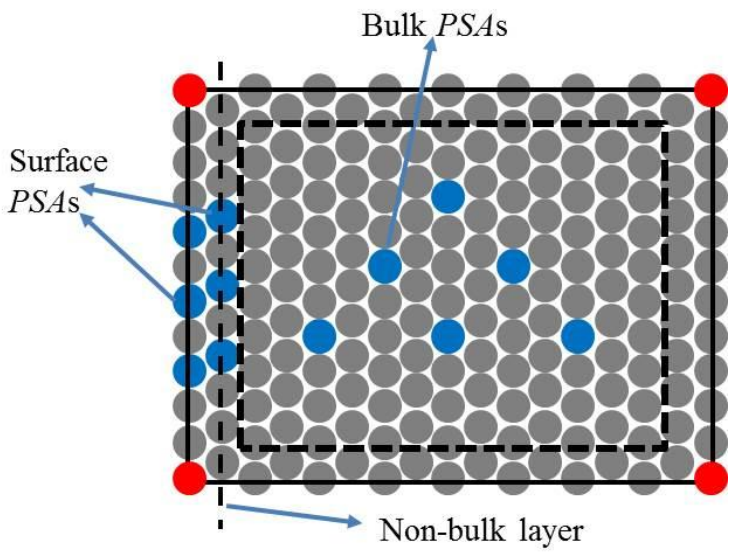

(d)

Figure 4 Employed $\mathrm{SR}^{\mathrm{MMM}}\left(\mathrm{SR}^{\mathrm{B}}+\mathrm{SR}^{\mathrm{S}}\right)$ in $2 \mathrm{D}$ for the selected 4-node quadrilateral element: $\mathrm{SR}^{\mathrm{MMM}}$ for an element that contains a corner atom (a); $\mathrm{SR}^{\mathrm{MMM}}$ for an element that contains only one surface edge (b); $\mathrm{SR}^{\mathrm{MMM}}$ for an element that contains a corner atom and non-bulk layers (dashed line) (c); $\mathrm{SR}^{\mathrm{MMM}}$ for an element that contains one surface edge and one non-bulk layer (d). Dashed rectangle represents the bulk region. $\mathrm{SR}^{\mathrm{B}}$ is employed for bulk non-sampling atoms (NSAs) and $\mathrm{SR}^{\mathrm{S}}$ for surface and non-bulk NSAs 


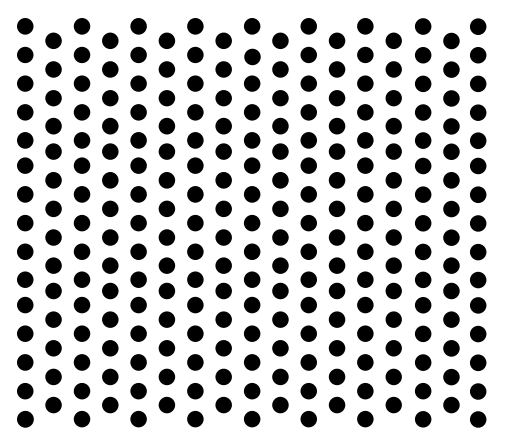

(a) Model A: $\mathbf{U}^{\mathrm{FA}}$

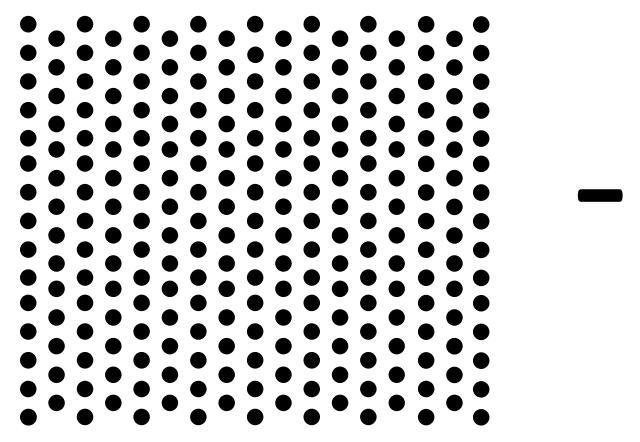

(a) Model A: $\mathbf{U}^{\mathrm{FA}}$

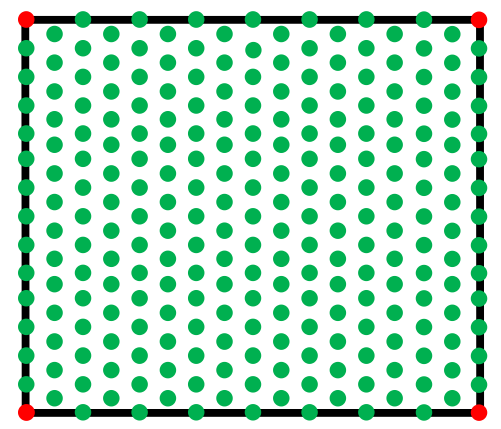

(c) Model C: $\mathbf{U}_{0 \mathrm{p} * \mathrm{~S}}^{\mathrm{MMM}}$

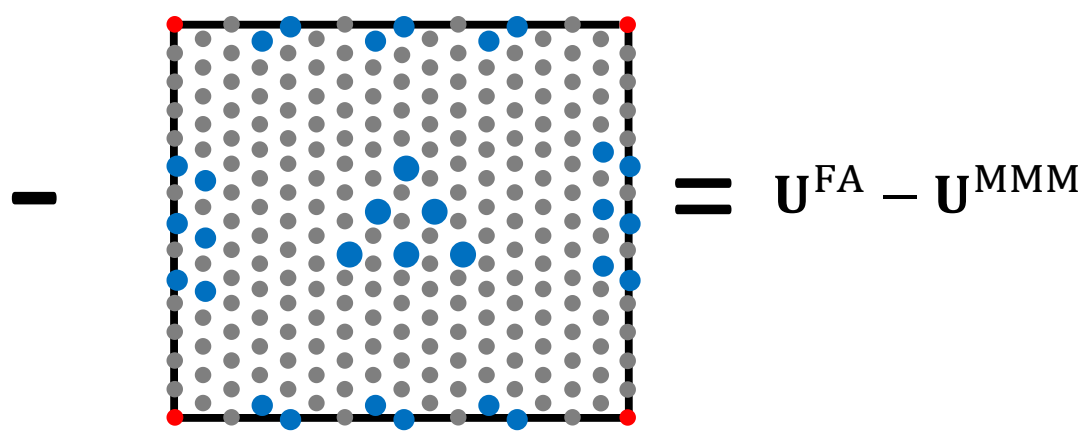

(b) Model B: $\mathbf{U}^{\mathrm{MMM}}$

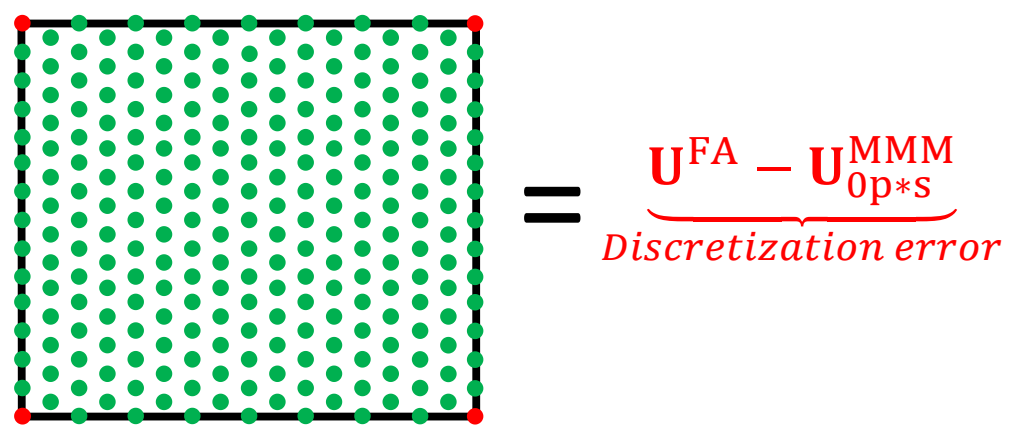

(c) Model C: $\mathbf{U}_{0 \mathrm{p} * \mathrm{~s}}^{\mathrm{MMM}}$

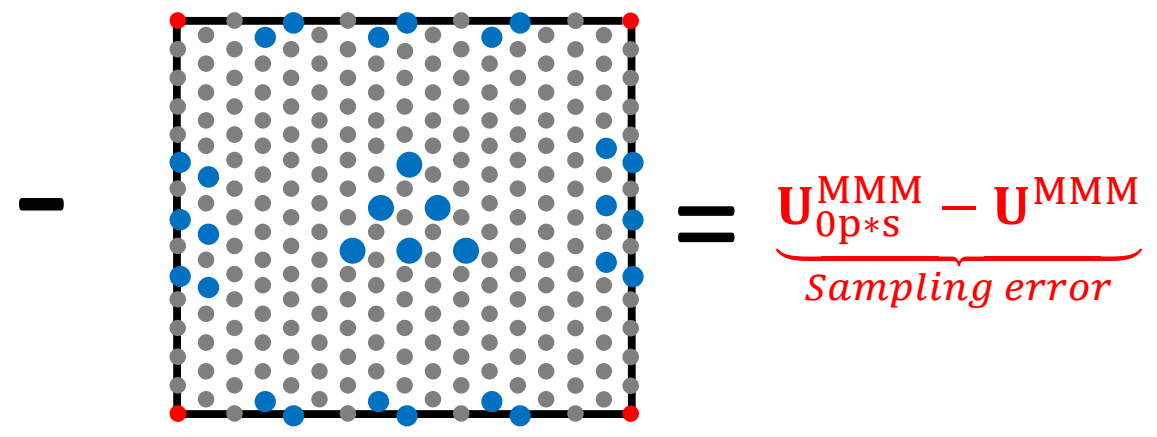

(b) Model B: $\mathbf{U}^{\mathrm{MMM}}$

Figure 5 Schematically demonstration of error sources and types: Model A: full atomistic model; Model B: a general MMM model with $\mathrm{SR}^{\mathrm{MMM}}$; Model C: a specifically designed model where the energy of each atom is explicitly considered. The distance between Modes A and B is the total error; distance between Models $\mathrm{A}$ and $\mathrm{C}$ is the discretization error; distance between Models $\mathrm{C}$ and $\mathrm{B}$ is the sampling error. Discretization error is fixed for a given mesh. 


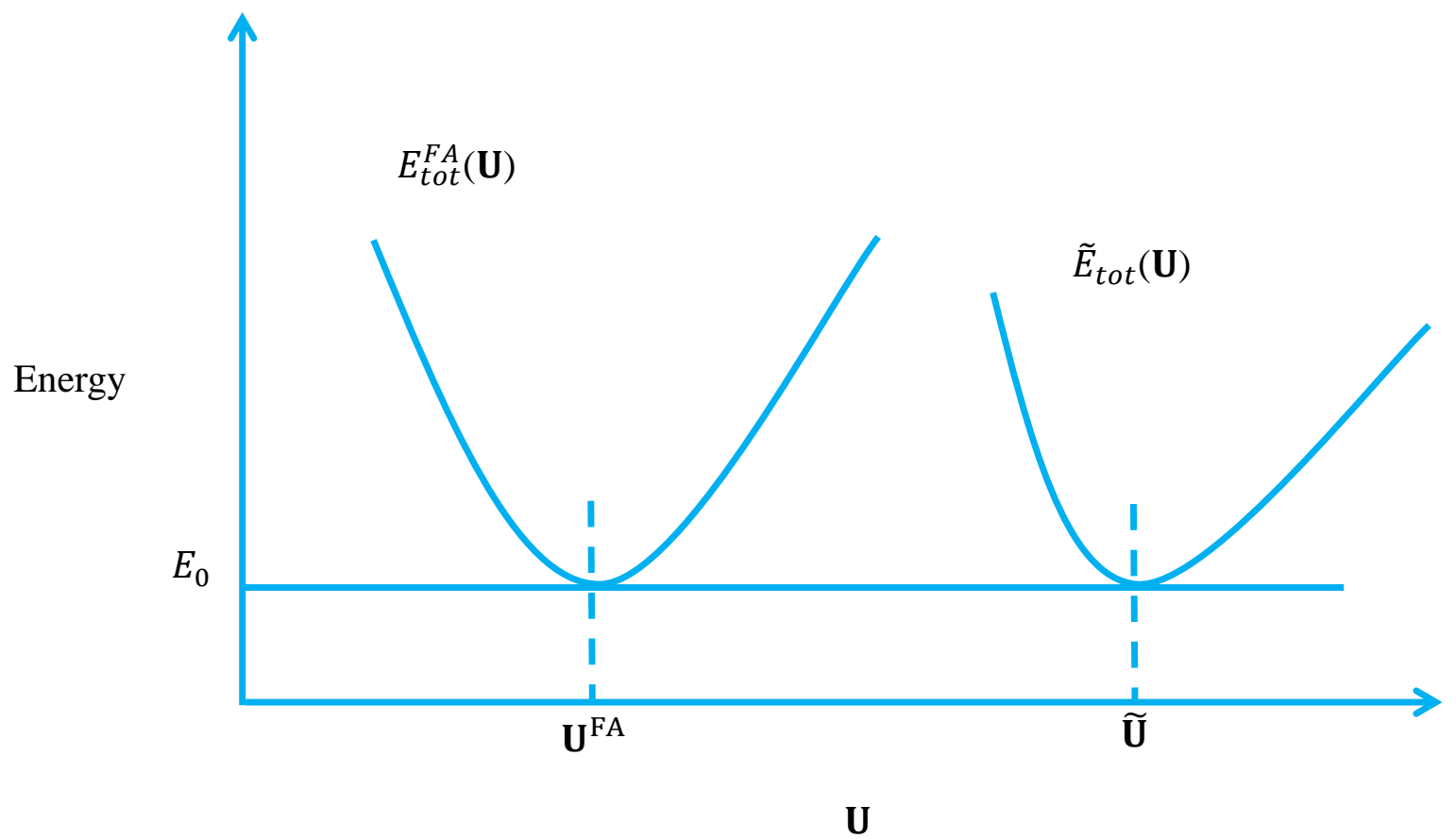

Figure 6 Schematically illustration of the possibility in an error estimator that the distance between different displacement fields could be very large even though they produce the same potential energy scalar $E_{0}: \mathbf{U}^{\mathrm{FA}}$ is from full atomistic simulation and $\widetilde{\mathbf{U}}$ from any other approximation. 


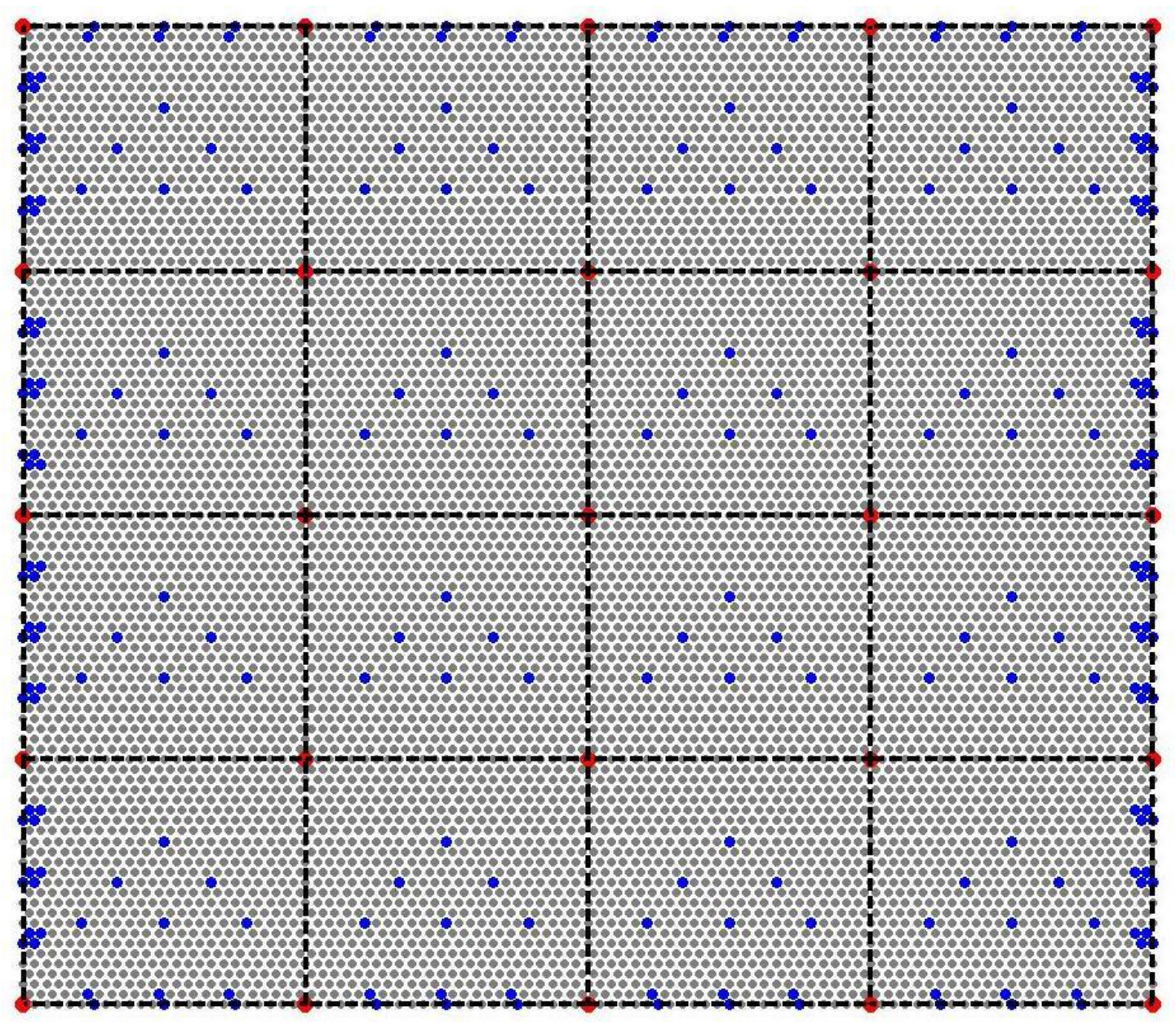

Figure 7 The employed 4-node quadrilateral mesh (dashed line) and summation rule $\mathrm{SR}^{\mathrm{MMM}}$ for the $2 \mathrm{D}$ surface relaxation problem: $\mathrm{SR}^{\mathrm{B}}$ for the bulk region and $\mathrm{SR}^{\mathrm{S}}$ for the surface and non-bulk regions 


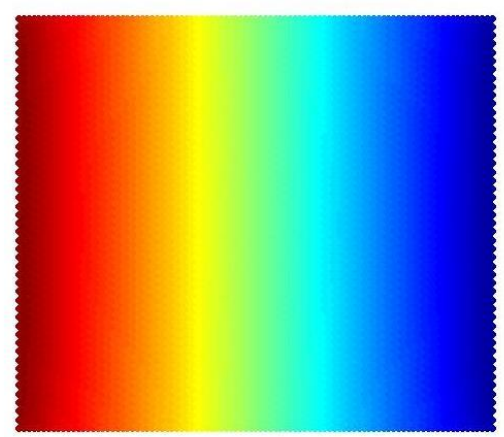

(a)

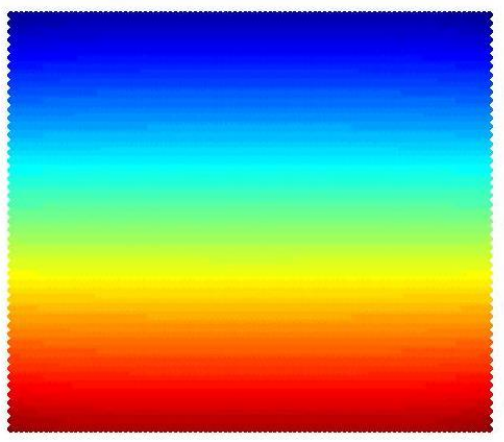

(c)

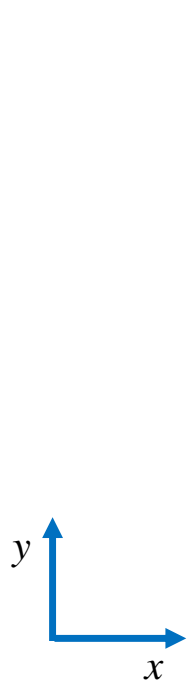

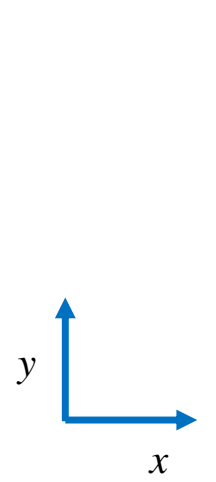

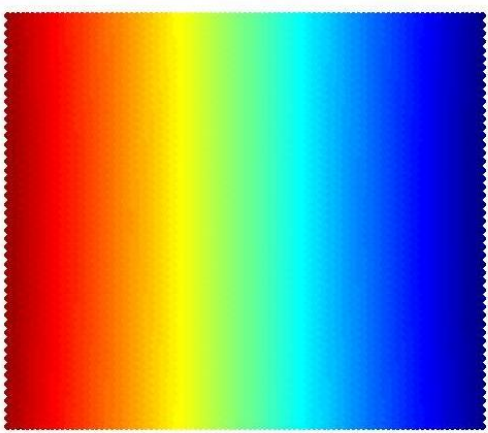

(b)

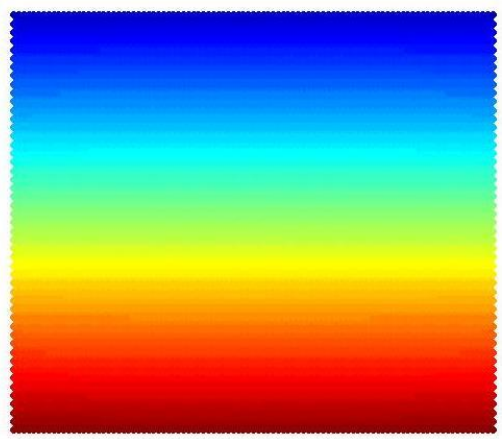

(d)
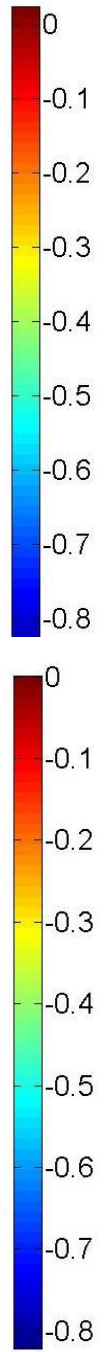

Figure 8 Comparison of displacement distributions from full atomistic (FA) calculation and MMM: the $x$-displacement distributions from FA (a) and MMM (b); the $y$-displacement distributions from FA (c) and MMM (d) 


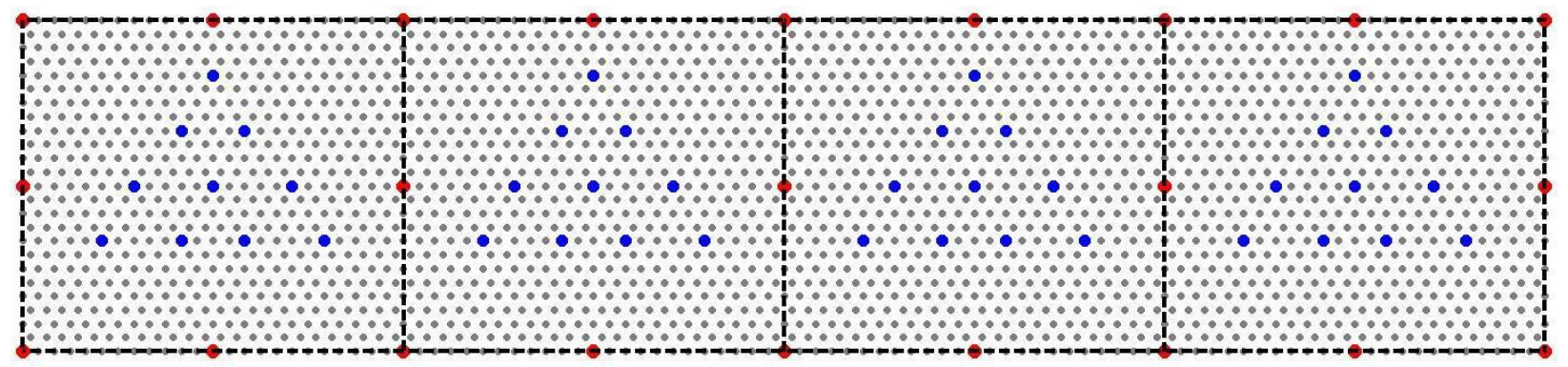

(a)

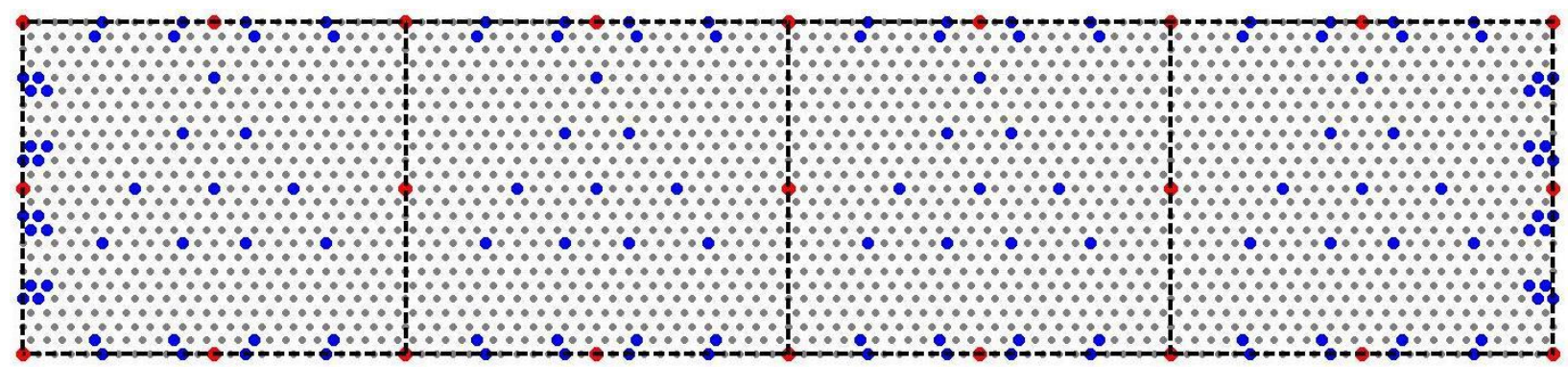

(b)

Figure 9 The employed 8-node quadratic quadrilateral mesh (dashed line) with $\mathrm{SR}^{\mathrm{B}}$ only (a) and with $\left(\mathrm{SR}^{\mathrm{S}}+\mathrm{SR}^{\mathrm{B}}\right)(\mathrm{b})$ for the $2 \mathrm{D}$ beam bending problem 


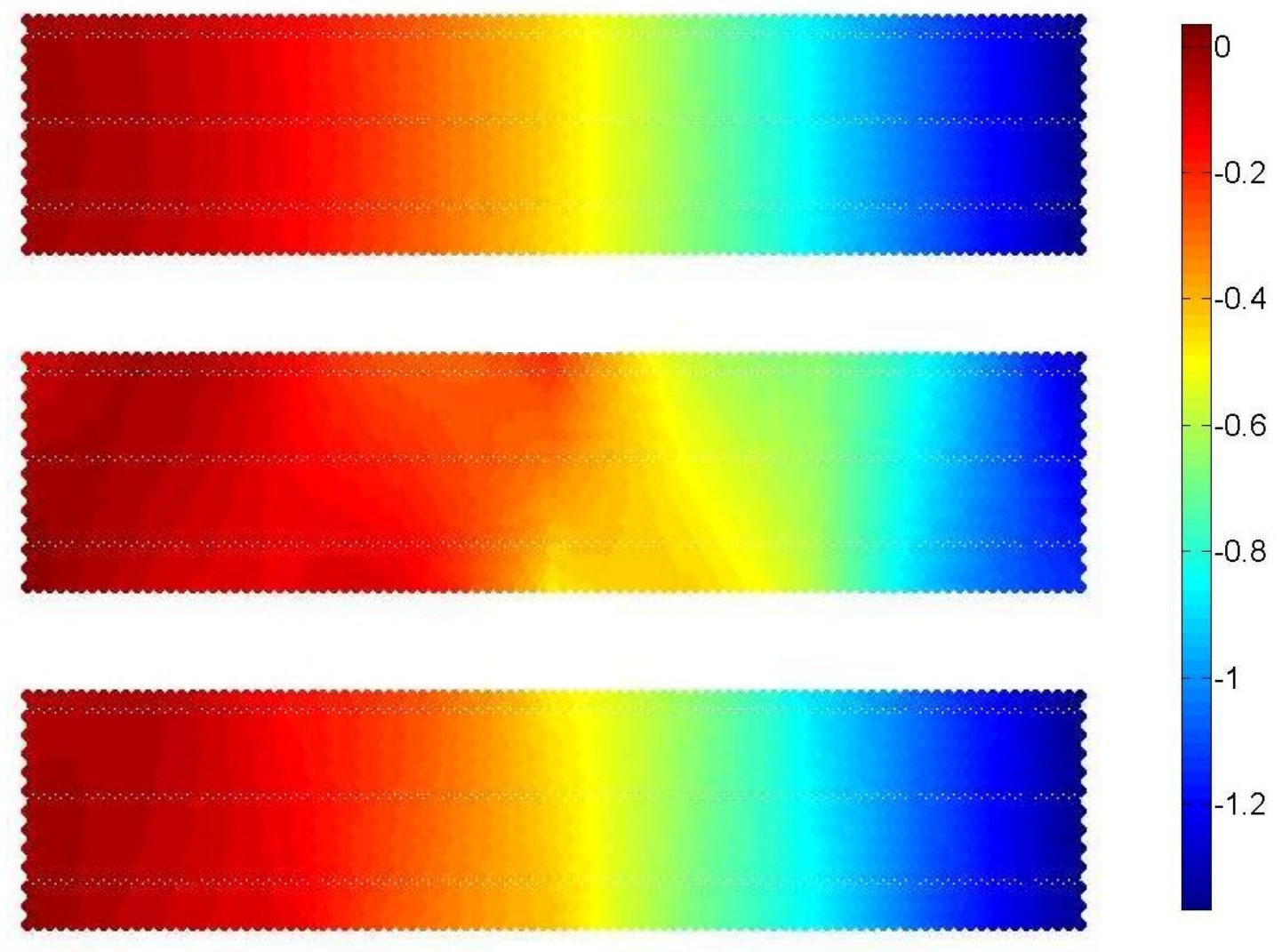

Figure 10 Comparison of vertical bending distributions from full atomistic (FA) (a), $\mathrm{SR}^{\mathrm{B}}$ only (b) and $\left(\mathrm{SR}^{\mathrm{S}}+\mathrm{SR}^{\mathrm{B}}\right)(\mathrm{c})$ 


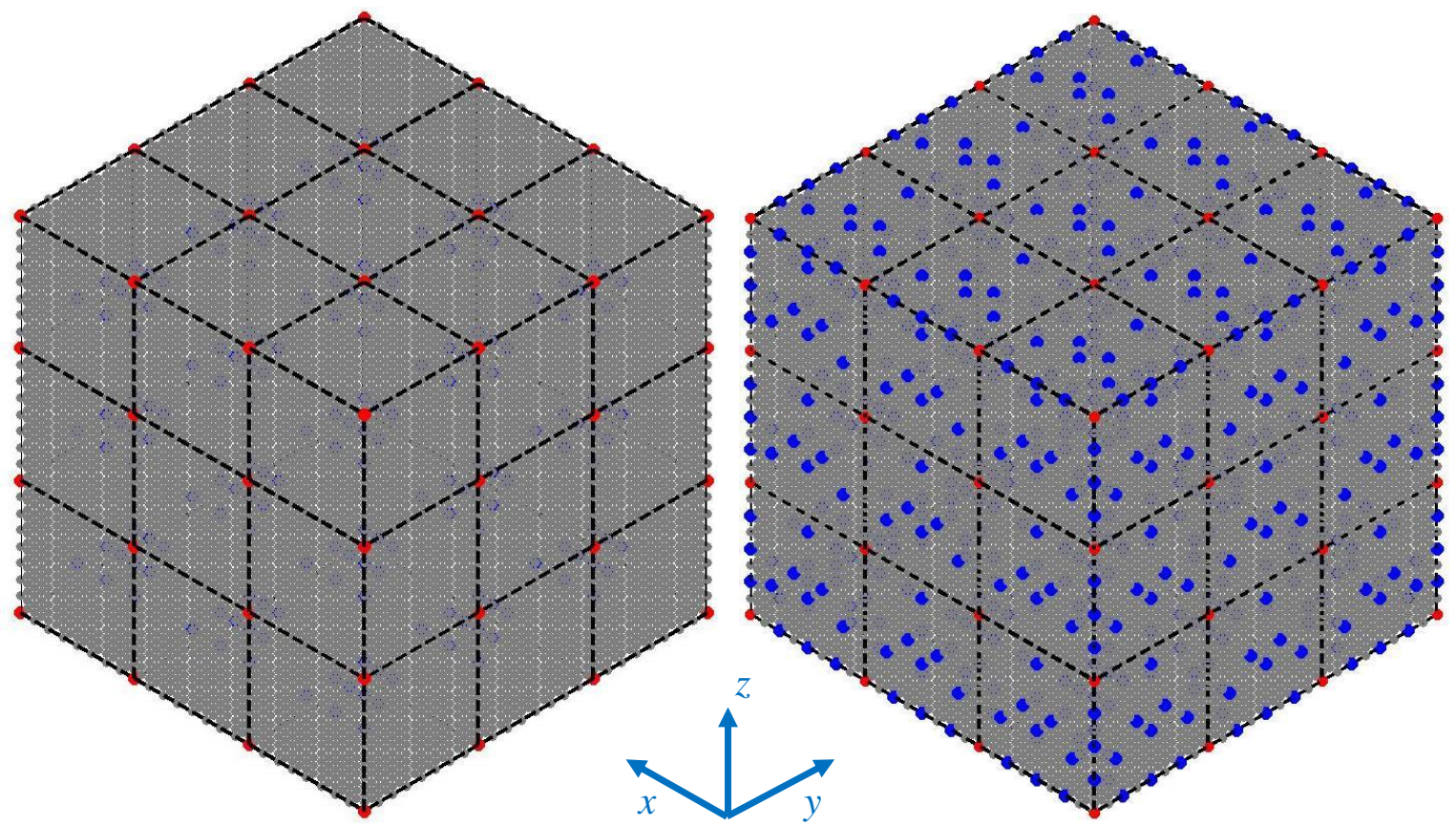

Figure 11 The employed 8-node hexahedral mesh (dashed line) for the $3 \mathrm{D}$ problem: $\mathrm{SR}^{\mathrm{B}}$ only is employed in (a) and $\left(\mathrm{SR}^{\mathrm{S}}+\mathrm{SR}^{\mathrm{B}}\right)$ is used in (b). The blue dots on the free surfaces and edges (b) shows the applied $\mathrm{SR}^{\mathrm{S}} ; \mathrm{SR}^{\mathrm{S}}$ and $\mathrm{SR}^{\mathrm{B}}$ are also utilized for the non-bulk and bulk layers, respectively, and are invisible 


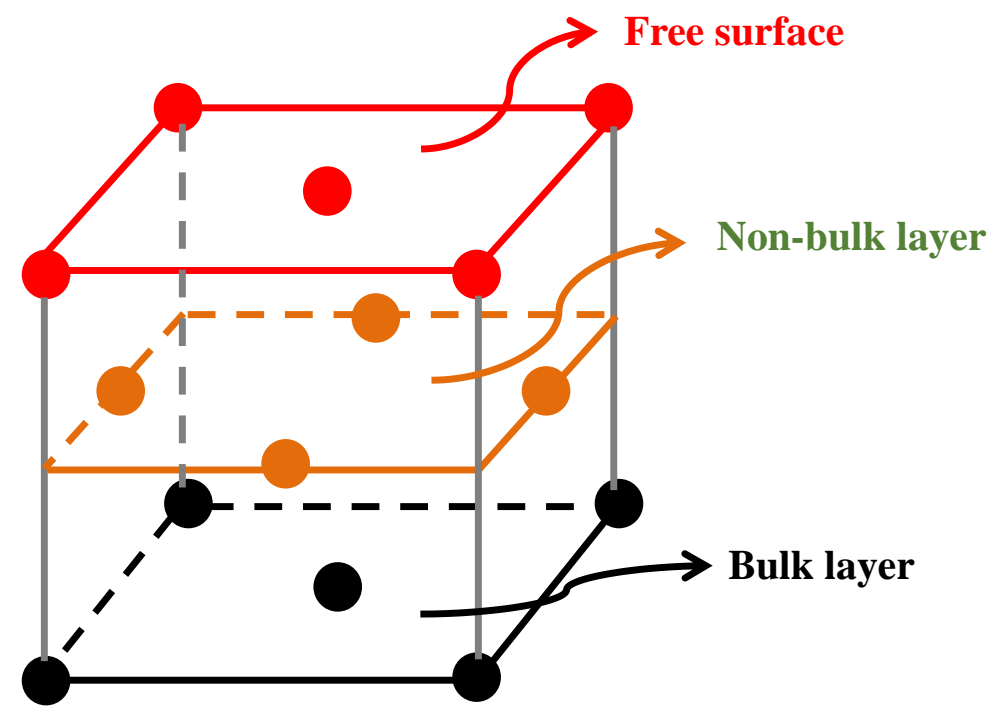

Figure 12 The respective free surface, non-bulk and bulk layers of the $3 \mathrm{D}$ example: $\mathrm{SR}^{\mathrm{S}}$ is applied to both free surface and non-bulk layers and $\mathrm{SR}^{\mathrm{B}}$ is employed for bulk layers 


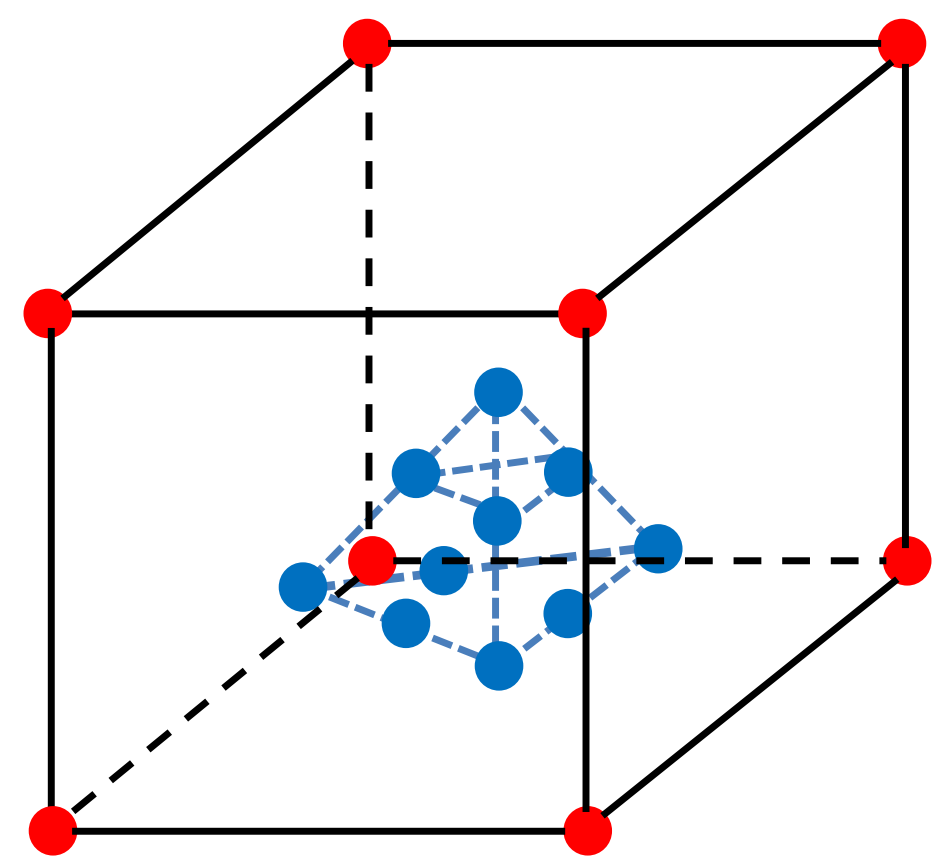

Figure 13 Schematically demonstration of the employed $\mathrm{SR}^{\mathrm{B}}$ with 8-node hexahedral element for bulk layers: red dots are the nodal atoms and blue dots represent the selected primary sampling atoms (PSAs) 

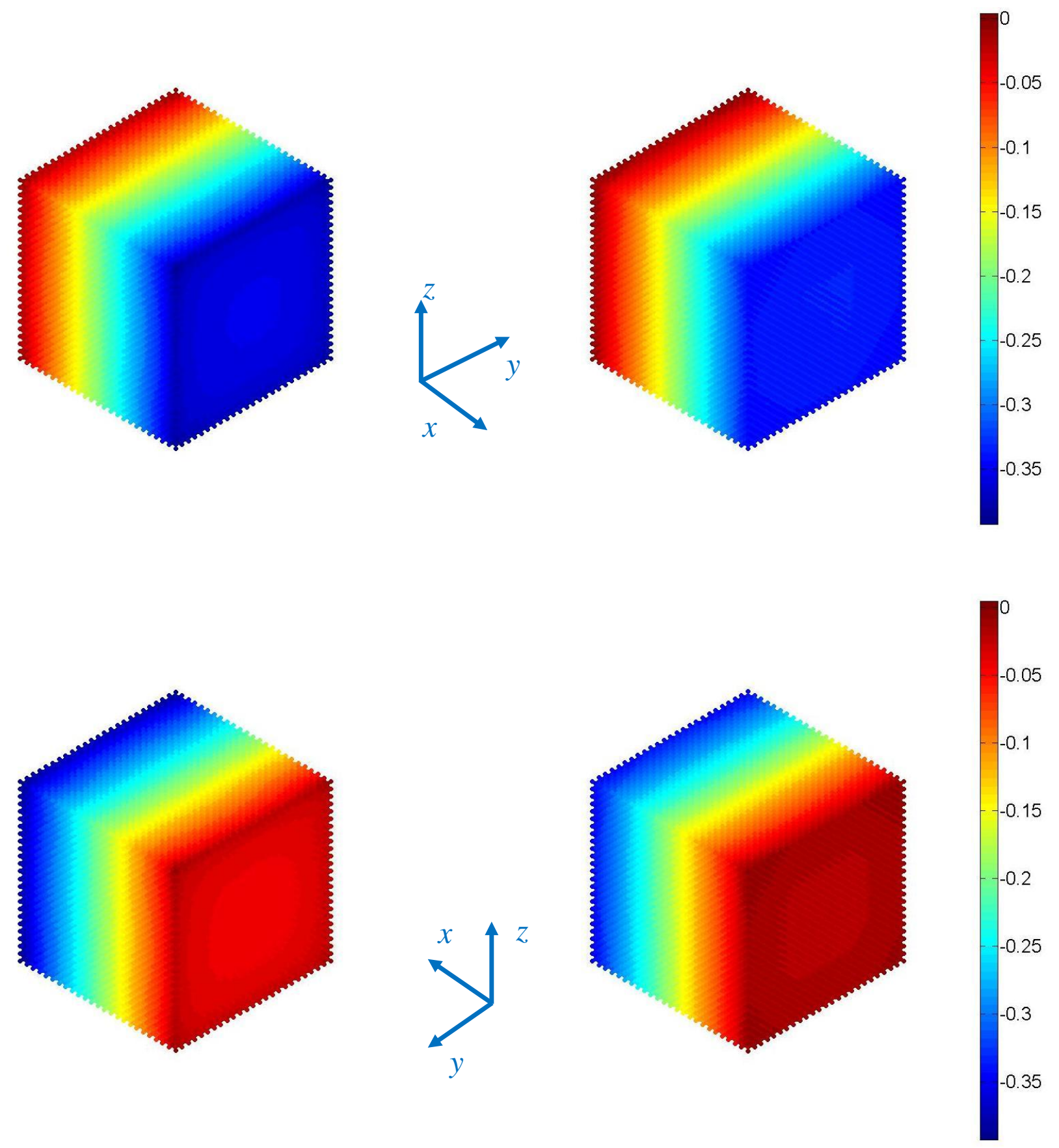

Figure 14 Comparison of $x$-displacement distributions on surfaces from full atomistics (FA) and MMM: $+x$-displacement from FA (a) and MMM (b); $-x$-displacement from FA (c) and MMM (d) 

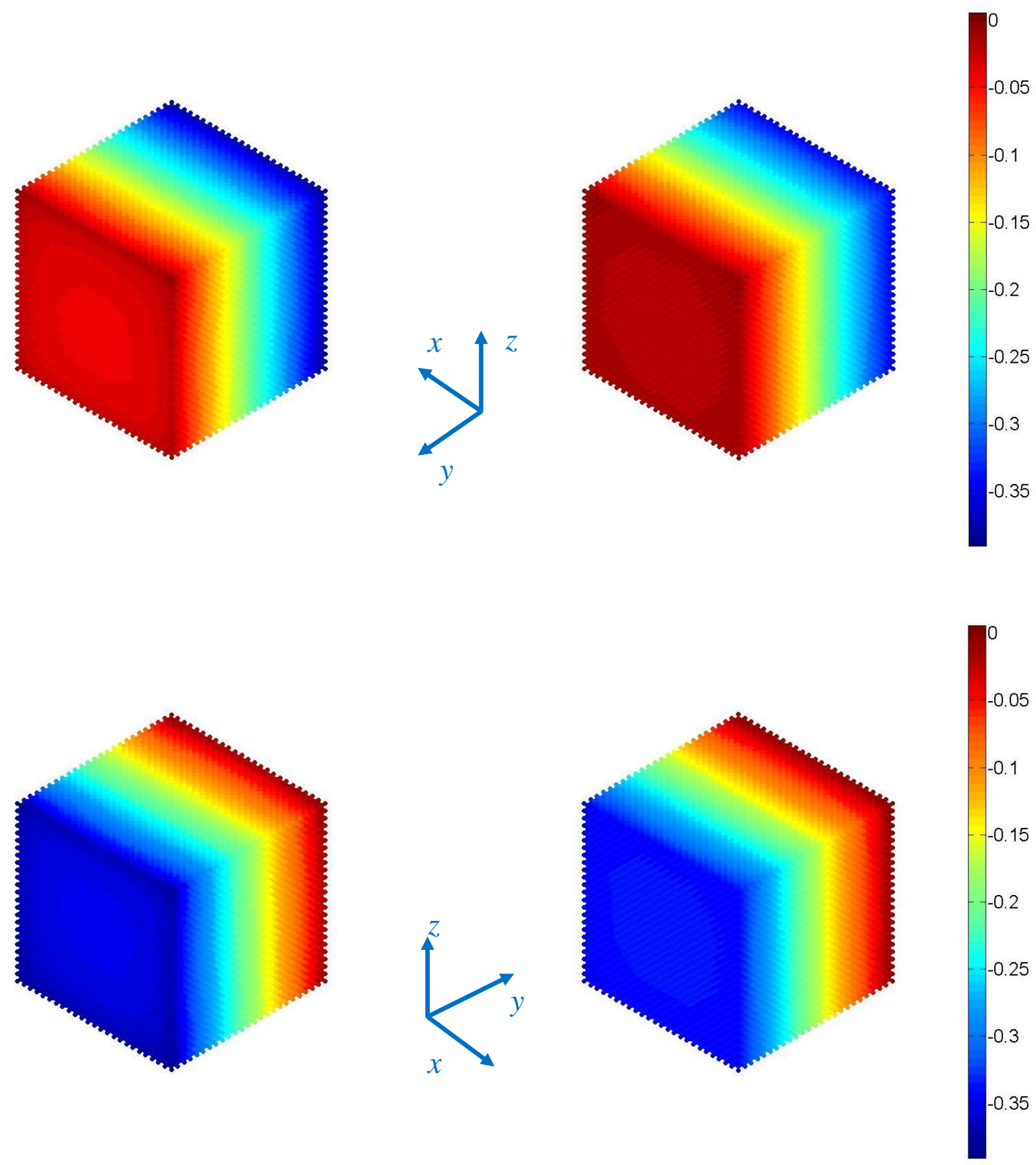

Figure 15 Comparison of $y$-displacement distributions on surfaces from full atomistics (FA) and MMM: $+y$-displacement from FA (a) and MMM (b); - $y$-displacement from FA (c) and MMM (d) 

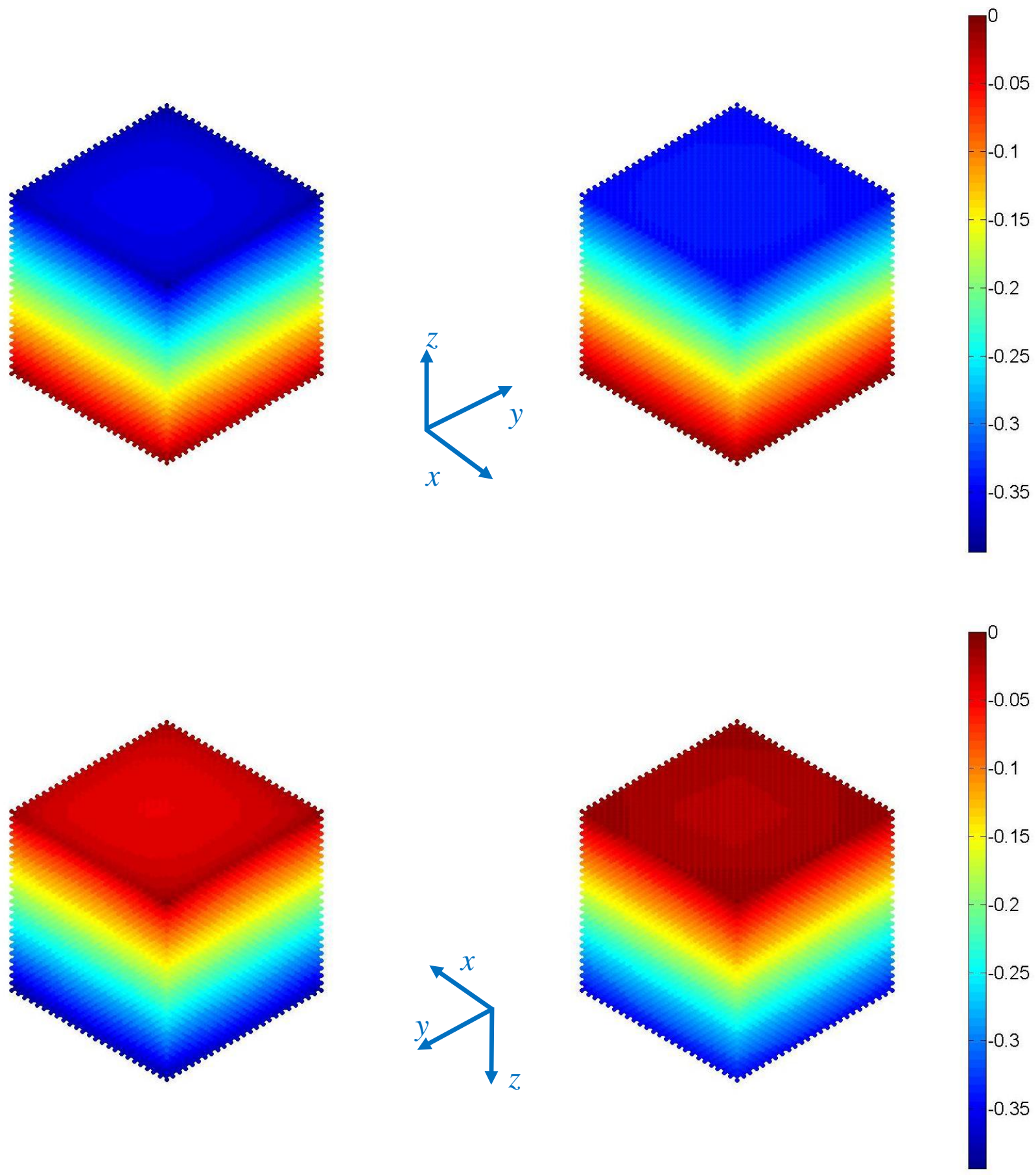

Figure 16 Comparison of $z$-displacement distributions on surfaces from full atomistics (FA) and MMM: +z-displacement from FA (a) and MMM (b); -z-displacement from FA (c) and MMM (d) 


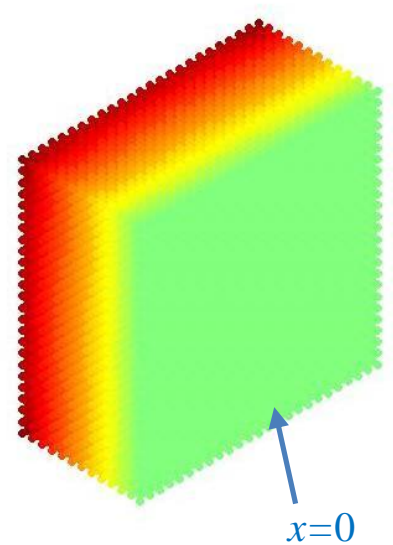

(a)

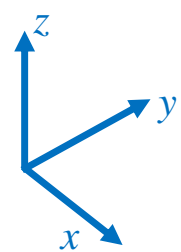

$x$

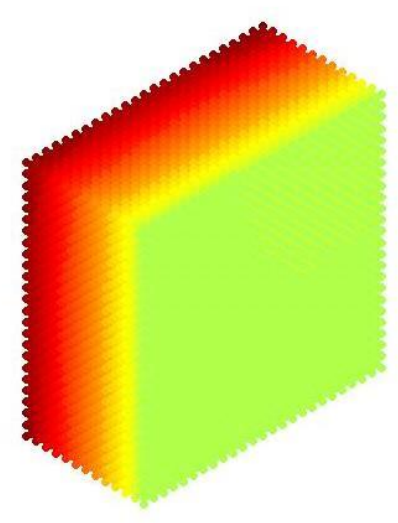

(b)

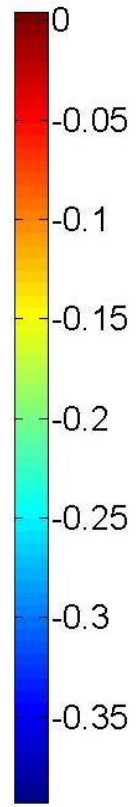

Figure 17 Comparison of $x$-displacement distributions from bulk along the plane $x=0$ from full atomistics (FA) (a) and MMM (b) 


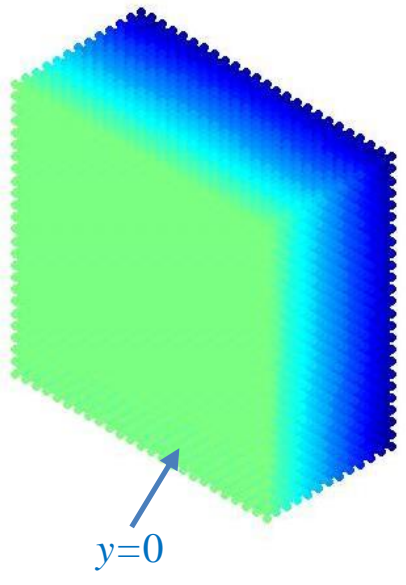

(a)

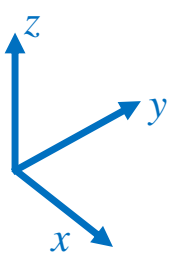

(b)

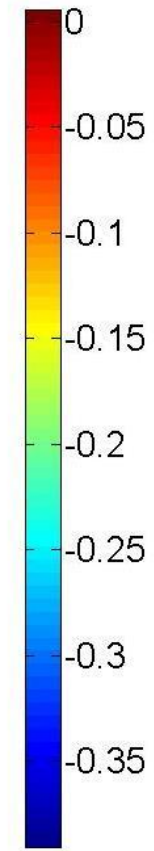

Figure 18 Comparison of $y$-displacement distributions from bulk along the plane $y=0$ from full atomistics (FA) (a) and MMM (b) 


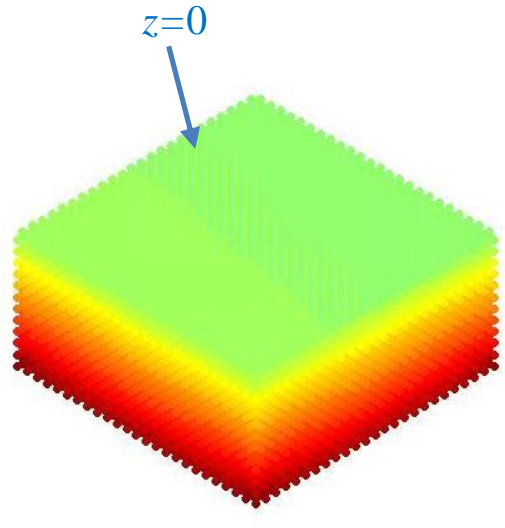

(a)

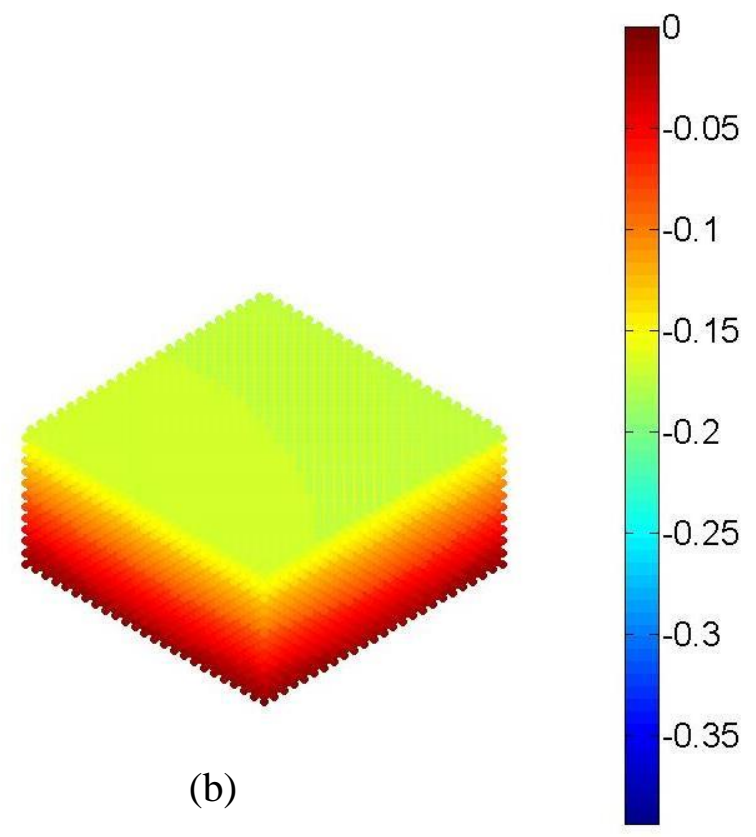

Figure 19 Comparison of $z$-displacement distributions from bulk along the plane $z=0$ from full atomistics (FA) (a) and MMM (b) 
Table 1: Normalized displacement comparison between full atomistic (FA) simulation and MMM calculation at the upper right corner and the middle of the edge at the right hand side

\begin{tabular}{lllll}
\hline \hline \multirow{2}{*}{ Method } & Corner & \multicolumn{3}{c}{ Middle of edge } \\
\cline { 2 - 5 } & $u_{x}$ & $u_{y}$ & $u_{x}$ & $u_{y}$ \\
\hline FA & 1 & 1 & 1 & 1 \\
MMM & 1.04 & 0.94 & 1.02 & 0.94 \\
\hline \hline
\end{tabular}

Table 2: Different types of errors in displacement and energy field in the 2D surface relaxation example with bilinear quadrilateral element

\begin{tabular}{lllll}
\hline \hline Summation rule & $e_{U}^{\text {total }}$ & $e_{U}^{\text {disc }}$ & $e_{U}^{\text {sam }}$ & $e_{E}^{\text {sam }}$ \\
\hline $\mathrm{SR}^{\mathrm{MMM}}\left(\mathrm{SR}^{\mathrm{B}}+\mathrm{SR}^{\mathrm{S}}\right)$ & $4.85 \%$ & $4.82 \%$ & $0.04 \%$ & $0.0006 \%$ \\
\hline \hline
\end{tabular}

Table 3: Different types of errors in displacement and energy field in the 2D beam bending example employing quadratic quadrilateral element

\begin{tabular}{lllll}
\hline \hline Summation rule & $e_{U}^{\text {total }}$ & $e_{U}^{\text {disc }}$ & $e_{U}^{\text {sam }}$ & $e_{E}^{\text {sam }}$ \\
\hline $\mathrm{SR}^{\mathrm{MMM}}\left(\mathrm{SR}^{\mathrm{B}}\right.$ only $)$ & $28.28 \%$ & $0.09 \%$ & $28.25 \%$ & $104.8 \%$ \\
\hline $\mathrm{SR}^{\mathrm{MMM}}\left(\mathrm{SR}^{\mathrm{B}}+\mathrm{SR}^{\mathrm{S}}\right)$ & $1.03 \%$ & $0.09 \%$ & $1.06 \%$ & $14.97 \%$ \\
& & & & \\
\hline \hline
\end{tabular}

Table 4: Normalized displacement comparison between full atomistic (FA) simulation and MMM calculation at corner, middle of edge and face center in the 3D example

\begin{tabular}{llllllllll}
\hline \hline \multirow{2}{*}{ Method } & \multicolumn{3}{c}{ Corner } & \multicolumn{3}{c}{ Middle of edge } & \multicolumn{3}{c}{ Face center } \\
\cline { 2 - 11 } & $u_{x}$ & $u_{y}$ & $u_{z}$ & $u_{x}$ & $u_{y}$ & $u_{z}$ & $u_{x}$ & $u_{y}$ & $u_{z}$ \\
\hline FA & 1 & 1 & 1 & 1 & 1 & 1 & 1 & 1 & 1 \\
MMM & 0.89 & 0.90 & 0.89 & 0.92 & 0.91 & 0.92 & 0.95 & 0.91 & 0.90 \\
\hline \hline
\end{tabular}

Table 5: Different types of errors in displacement and energy field in the 3D example with hexahedral element

\begin{tabular}{lllll}
\hline \hline Summation rule & $e_{U}^{\text {total }}$ & $e_{U}^{\text {disc }}$ & $e_{U}^{\text {sam }}$ & $e_{E}^{\text {sam }}$ \\
\hline $\mathrm{SR}^{\mathrm{MMM}}\left(\mathrm{SR}^{\mathrm{B}}\right.$ only $)$ & $34.34 \%$ & $10.30 \%$ & $44.53 \%$ & $1.02 \%$ \\
\hline $\mathrm{SR}^{\mathrm{MMM}}\left(\mathrm{SR}^{\mathrm{B}}+\mathrm{SR}^{\mathrm{S}}\right)$ & $9.92 \%$ & $10.30 \%$ & $0.71 \%$ & $0.086 \%$ \\
\hline \hline
\end{tabular}

